\title{
Phenomenology of Higgs bosons in the Zee model
}

\author{
Shinya Kanemura* \\ Institut für Theoretische Physik, Universität Karlsruhe, D-76128 Karlsruhe, Germany \\ and Physics and Astronomy Department, Michigan State University, East Lansing, Michigan 48824-1116 \\ Takashi Kasai ${ }^{\dagger}$ \\ Theory Group, KEK, Tsukuba, Ibaraki, 305-0801 Japan \\ and Department of Accelerator Science, The Graduate University for Advanced Studies, Tsukuba, Ibaraki, 305-0801 Japan \\ Guey-Lin Lin \\ National Chiao Tung University, Hsinchu 300, Taiwan \\ Yasuhiro Okada ${ }^{\S}$ \\ Theory Group, KEK, Tsukuba, Ibaraki, 305-0801 Japan \\ and Department of Particle and Nuclear Physics, The Graduate University for Advanced Studies, Tsukuba, Ibaraki, $305-0801$ Japan \\ Jie-Jun Tsengl\| \\ National Chiao Tung University, Hsinchu 300, Taiwan \\ C.-P. Yuan ${ }^{\text {II }}$ \\ Physics and Astronomy Department, Michigan State University, East Lansing, Michigan 48824-1116 \\ and Theory Division, CERN, CH-1211, Geneva, Switzerland \\ (Received 2 December 2000; published 7 August 2001)
}

\begin{abstract}
To generate small neutrino masses radiatively, the Zee model introduces two Higgs doublets and one weak-singlet charged Higgs boson to its Higgs sector. From analyzing the renormalization group equations, we determine the possible range of the lightest $C P$-even Higgs boson $(h)$ mass and the Higgs boson self-couplings as a function of the cutoff scale beyond which either some of the coupling constants are strong enough to invalidate the perturbative analysis or the stability of the electroweak vacuum is no longer guaranteed. Using the results obtained from the above analysis, we find that the singlet charged Higgs boson can significantly modify the partial decay width of $h \rightarrow \gamma \gamma$ via radiative corrections, and its collider phenomenology can also be drastically different from that of the charged Higgs bosons in the usual two-Higgs-doublet models.
\end{abstract}

DOI: 10.1103/PhysRevD.64.053007

\section{INTRODUCTION}

There is increasing evidence for neutrino oscillations from atmospheric and solar neutrino data [1]. If this is a correct interpretation, the standard model (SM) has to be extended to incorporate the small masses of the neutrinos suggested by the data. There have been several ideas proposed in the literature to generate small neutrino masses. The Zee model is one such attempt [2-6]. In this model, all flavor neutrinos are massless at the tree level, and their small masses are induced radiatively through one-loop diagrams. For such a mass-generation mechanism to work, it is necessary to extend the Higgs sector of the SM to contain at least two weak-doublet fields and one weak-singlet charged scalar field. Although some studies have been done to examine the interaction of the leptons and the Higgs bosons in the Zee model [7], the scalar (Higgs) sector of the model remains

\footnotetext{
*Email address: kanemu@ particle.physik.uni-karlsruhe.de

${ }^{\dagger}$ Email address: kasai@ post.kek.jp

*Email address: glin@cc.nctu.edu.tw

${ }^{\S}$ Email address: yasuhiro.okada@kek.jp

"Email address: u8627512@cc.nctu.edu.tw

ITEmail address: yuan@pa.msu.edu
}

PACS number(s): 13.15.+g, 14.80.Cp

unexplored in detail. In this paper we study the Higgs sector of the Zee model to clarify its impact on the Higgs search experiments, at the CERN $e^{+} e^{-}$collider LEP-II, run II of the Fermilab Tevatron, the CERN Large Hadron Collider (LHC), or future linear colliders (LC's).

Experimental search for the Higgs boson has been continued at the CERN LEP and the Fermilab Tevatron experiments. In the LEP-II experiments, the Higgs boson with mass less than about $110 \mathrm{GeV}$ has been excluded if its production cross section and decay modes are similar to those of the SM Higgs boson [8]. Run II of the Tevatron can be sensitive to a SM-like Higgs boson with mass up to about 180 $\mathrm{GeV}$, provided that the integrated luminosity of the collider is large enough (about $30 \mathrm{fb}^{-1}$ ) [9]. Furthermore, the primary goal of the CERN LHC experiments is to guarantee the discovery of a SM-like Higgs boson with mass as large as about $1 \mathrm{TeV}[10]$, which is the upper bound of the SM Higgs boson mass. (For a Higgs boson mass beyond this value, the SM is no longer a consistent low energy theory.)

When the Higgs boson is discovered, its mass and various decay properties will be measured to test the SM and to distinguish models of new physics at high energy scales. For example, the allowed mass range of the lightest $C P$-even Higgs boson $(h)$ can be determined by demanding that the considered theory be a valid effective theory all the way up 
to some cutoff energy scale $(\Lambda)$. For $\Lambda=10^{19} \mathrm{GeV}$ (i.e., the Planck scale), the lower and upper bounds of the SM Higgs boson masses are 137 and $175 \mathrm{GeV}$, respectively [11]. The Higgs boson mass bounds for the two-Higgs-doublet model (THDM) were also investigated $[12,13]$ with and without including the soft-breaking term with respect to the discrete symmetry that protects the natural flavor conservation. It was found in Ref. [13] that the lower bound of the lightest $\mathrm{CP}$ even Higgs boson is about $100 \mathrm{GeV}$ in the decoupling regime where only one neutral Higgs boson is light as compared to the other physical states of the Higgs bosons.

The Higgs sector of the Zee model is similar to that of the THDM except for the existence of an additional weak-singlet charged Higgs field, so that the physical scalar bosons include two $C P$-even, one $C P$-odd, and two pairs of charged Higgs bosons. In this paper, we shall first determine the upper and lower bounds for the lightest $C P$-even Higgs boson mass $\left(m_{h}\right)$ as a function of the cutoff scale $\Lambda$ of the Zee model, using renormalization group equations (RGE's). ${ }^{1} \mathrm{We}$ show that the upper and lower mass bounds for $h$ are almost the same as those in the THDM. We also study the possible range of the Higgs boson self-coupling constants at the electroweak scale as a function of $\Lambda$. By using these results, we examine effects of the additional loop contribution of the singlet charged Higgs boson to the partial decay width of $h$ $\rightarrow \gamma \gamma$. We show that, by taking $\Lambda=10^{19} \mathrm{GeV}$, the deviation of the decay width from the SM prediction can be about $-20 \%$ or nearly $+10 \%$ for $m_{h}$ between 125 and $140 \mathrm{GeV}$ when the mass of the isospin singlet charged Higgs boson is taken to be around $100 \mathrm{GeV}$. The magnitude of the deviation becomes larger for lower cutoff scales and smaller masses of the singlet charged Higgs boson. If we choose $\Lambda=10^{4} \mathrm{GeV}$ and the singlet charged Higgs boson mass to be $100 \mathrm{GeV}$, the positive deviation can be greater than $+30 \%(+40 \%)$ for $m_{h}=125 \mathrm{GeV}(140 \mathrm{GeV})$. Such a deviation from the SM prediction could be tested at the LHC, the $e^{+} e^{-} \mathrm{LC}$, and the $\gamma \gamma$ option of the LC [15-17]. We also discuss the phenomenology of the singlet charged Higgs boson at present and future collider experiments; it is found to be completely different from that of ordinary THDM-like charged Higgs bosons. To detect such a charged Higgs boson at LEP-II experiments, experimentalists have to search for their data sample with $e^{ \pm}$or $\mu^{ \pm}$plus missing energy, in contrast to the usual detection channels: either $\tau \nu$ or $c s$ decay modes.

This paper is organized as follows. In Sec. II, we introduce the Higgs sector of the Zee model and review the neutrino masses and mixings in this model that are consistent with the atmospheric and solar neutrino observations. Numerical results for the possible range of the mass and coupling constants of the Higgs bosons are given in Sec. III. In Sec. IV, we discuss the one-loop effect of the extra Higgs bosons in the Zee model on the partial decay width of $h$ $\rightarrow \gamma \gamma$ and its impacts on the neutral Higgs boson search at high energy colliders. The phenomenology of the charged

\footnotetext{
${ }^{1}$ For the model with seesaw mechanism for neutrino mass generation the Higgs boson mass bound has been studied as a function of cutoff scale in Ref. [14].
}

Higgs boson that comes from the additional singlet field is discussed in Sec. V. In Sec. VI, we present additional discussion and conclusions. Relevant RGE's for the Zee model are given in the Appendix.

\section{ZEE MODEL}

To generate small neutrino mass radiatively, the Zee model contains an $\mathrm{SU}(2)_{L}$ singlet charged scalar field $\omega^{-}$, in addition to two $\mathrm{SU}(2)_{L}$ doublet fields $\phi_{1}$ and $\phi_{2}$. The Zeemodel Lagrangian is written as

$$
\mathcal{L}=\mathcal{L}_{\text {kin }}+\mathcal{L}_{l l \omega}+\mathcal{L}_{\text {Yukawa }}-V\left(\phi_{1}, \phi_{2}, \omega^{-}\right)
$$

where

$$
\begin{aligned}
\mathcal{L}_{\text {kin }}= & \left|D_{\mu} \phi_{1}\right|^{2}+\left|D_{\mu} \phi_{2}\right|^{2}+\left|D_{\mu} \omega^{-}\right|^{2}+i \overline{q_{L}} \gamma^{\mu} D_{\mu} q_{L} \\
& +i \overline{u_{R}} \gamma^{\mu} D_{\mu} u_{R}+i \overline{d_{R}} \gamma^{\mu} D_{\mu} d_{R}+i \overline{l_{L}} \gamma^{\mu} D_{\mu} l_{L} \\
& +\overline{i e_{R}} \gamma^{\mu} D_{\mu} e_{R}+{ }_{a=\mathrm{SU}(3), \mathrm{SU}(2), U(1)} \overline{4} F_{\mu \nu}^{a^{2}}, \\
\mathcal{L}_{l l \omega}= & f_{i j} \overline{l_{i_{L}}}\left(i \tau_{2}\right)\left(l_{j_{L}}{ }^{C} \omega^{-}+f_{i j} \overline{l_{i}}{ }^{C}\left(i \tau_{2}\right) l_{j_{L}} \omega^{+},\right.
\end{aligned}
$$

where $i, j(=1,2,3)$ are the generation indices, and

$$
\begin{aligned}
V\left(\phi_{1}, \phi_{2}, \omega^{-}\right)= & m_{1}^{2}\left|\phi_{1}\right|^{2}+m_{2}^{2}\left|\phi_{2}\right|^{2}+m_{0}^{2}\left|\omega^{-}\right|^{2}-m_{3}^{2}\left(\phi_{1}^{\dagger} \phi_{2}\right. \\
& \left.+\phi_{2}^{\dagger} \phi_{1}\right)-\mu \widetilde{\phi}_{1}^{T} i \tau_{2} \widetilde{\phi}_{2} \omega^{-}+\mu \phi_{2}^{T} i \tau_{2} \phi_{1} \omega^{+} \\
& +\frac{1}{2} \lambda_{1}\left|\phi_{1}\right|^{4}+\frac{1}{2} \lambda_{2}\left|\phi_{2}\right|^{4}+\lambda_{3}\left|\phi_{1}\right|^{2}\left|\phi_{2}\right|^{2} \\
& +\lambda_{4}\left|\phi_{1}^{\dagger} \phi_{2}\right|^{2}+\frac{\lambda_{5}}{2}\left[\left(\phi_{1}^{\dagger} \phi_{2}\right)^{2}+\left(\phi_{2}^{\dagger} \phi_{1}\right)^{2}\right] \\
& +\sigma_{1}\left|\omega^{-}\right|^{2}\left|\phi_{1}\right|^{2}+\sigma_{2}\left|\omega^{-}\right|^{2}\left|\phi_{2}\right|^{2} \\
& +\frac{1}{4} \sigma_{3}\left|\omega^{-}\right|^{4} .
\end{aligned}
$$

In the above equations, $q_{L}$ is the left-handed quark doublet with an implicit generation index while $u_{R}$ and $d_{R}$ denote the right-handed singlet quarks. Similarly, $l_{L}$ and $e_{R}$ denote the left-handed and right-handed leptons in three generations. The charge conjugation of a fermion field is defined as $\psi^{C}$ $\equiv C \bar{\psi}^{T}$, where $C$ is the charge conjugation matrix $\left(C^{-1} \gamma^{\mu} C=-\gamma^{\mu T}\right)$ with the superscript $T$ indicating the transpose of a matrix. Also,

$$
\phi_{m}=\left(\begin{array}{c}
\phi_{m}^{0} \\
\phi_{m}^{-}
\end{array}\right)
$$

and $\widetilde{\phi}_{m} \equiv\left(i \tau_{2}\right) \phi_{m}^{*}$ with $m=1,2$. Without loss of generality, we have taken the antisymmetric matrix $f_{i j}$ and the coupling $\mu$ to be real in Eqs. (3) and (4). In order to suppress flavor changing neutral current at the tree level, a discrete symmetry, with $\phi_{1} \rightarrow \phi_{1}, \phi_{2} \rightarrow-\phi_{2}, \omega^{+} \rightarrow+\omega^{+}$, is imposed on the Higgs sector of the Lagrangian, which is broken softly by only the $m_{3}^{2}$ term and the $\mu$ term. Under the discrete symmetry there are two possible Yukawa interactions; that is, for type I, 


$$
\begin{aligned}
\mathcal{L}_{\text {Yukawa } I}= & \overline{d_{R_{i}}}\left(y_{D} V_{\text {CKM }}^{\dagger}\right)_{i j} \widetilde{\phi}_{2}^{\dagger} q_{L_{j}}+\overline{u_{R_{i}}}\left(y_{U}\right)_{i i} \phi_{2}^{\dagger} q_{L_{i}} \\
& +\overline{e_{R_{i}}}\left(y_{E}\right)_{i i} \widetilde{\phi}_{2}^{\dagger} l_{L_{i}}+\text { H.c. },
\end{aligned}
$$

and, for type II,

$$
\begin{aligned}
\mathcal{L}_{\text {Yukawa II }}= & \overline{d_{R_{i}}}\left(y_{D} V_{\mathrm{CKM}}^{\dagger}\right)_{i j} \widetilde{\phi}_{1}^{\dagger} q_{L_{j}}+\overline{u_{R_{i}}}\left(y_{U}\right)_{i i} \phi_{2}^{\dagger} q_{L_{i}} \\
& +\overline{e_{R_{i}}}\left(y_{E}\right)_{i i} \widetilde{\phi}_{1}^{\dagger} l_{L_{i}}+\text { H.c. },
\end{aligned}
$$

where $y_{U}, y_{D}, y_{E}$ are diagonal Yukawa matrices and $V_{\mathrm{CKM}}$ is the Cabibbo-Kobayashi-Maskawa (CKM) matrix. Later, we shall keep only the top Yukawa coupling constants $y_{t}$ $=\left(y_{U}\right)_{33}$ in our numerical evaluation of the RGE's. ${ }^{2}$ In that case, there is no difference between the Yukawa couplings of the type-I and type-II models. Finally, for simplicity, we assume that all $\lambda_{i}$ and $m_{i}^{2}$ are real parameters.

Let us now discuss the Higgs sector. The $\mathrm{SU}(2)_{L}$ $\times U(1)_{Y}$ symmetry is broken to $U(1)_{e m}$ by $\left\langle\phi_{1}\right\rangle$ and $\left\langle\phi_{2}\right\rangle$, the vacuum expectation values of $\phi_{1}$ and $\phi_{2}$. (They are assumed to be real so that there is no spontaneous $C P$ violation.) The number of physical Higgs bosons are two $C P$-even Higgs bosons $(H, h)$, one $C P$-odd Higgs boson $(A)$, and two pairs of charged Higgs bosons $\left(S_{1}^{ \pm}, S_{2}^{ \pm}\right)$. We take a convention of $m_{H}>m_{h}$ and $m_{S_{1}}>m_{S_{2}}$. In the basis where two Higgs doublets are rotated by the angle $\beta$, with $\tan \beta=\left\langle\phi_{2}^{0}\right\rangle /\left\langle\phi_{1}^{0}\right\rangle$, the mass matrices for the physical states of the Higgs bosons are given by

$$
M_{N}^{2}=\left[\begin{array}{cc}
\left(\lambda_{1} \cos ^{4} \beta+\lambda_{2} \sin ^{4} \beta+\frac{\lambda}{2} \sin ^{2} 2 \beta\right) v^{2} & \left(\lambda_{2} \sin ^{2} \beta-\lambda_{1} \cos ^{2} \beta+\lambda \cos 2 \beta\right) \frac{\sin 2 \beta}{2} v^{2} \\
\left(\lambda_{2} \sin ^{2} \beta-\lambda_{1} \cos ^{2} \beta+\lambda \cos 2 \beta\right) \frac{\sin 2 \beta}{2} v^{2} & M^{2}+\left(\lambda_{1}+\lambda_{2}-2 \lambda\right) \frac{\sin ^{2} 2 \beta}{4} v^{2}
\end{array}\right]
$$

for $C P$-even Higgs bosons,

$$
M_{A}^{2}=M^{2}-\lambda_{5} v^{2}
$$

for $\mathrm{CP}$-odd Higgs bosons, and

$$
M_{S}^{2}=\left[\begin{array}{cc}
M^{2}-\frac{\lambda_{4}+\lambda_{5}}{2} v^{2} & -\frac{\mu v}{\sqrt{2}} \\
-\frac{\mu v}{\sqrt{2}} & m_{0}^{2}+\left(\frac{\sigma_{1}}{2} \cos ^{2} \beta+\frac{\sigma_{2}}{2} \sin ^{2} \beta\right) v^{2}
\end{array}\right]
$$

for charged Higgs bosons. Here, $\lambda \equiv \lambda_{3}+\lambda_{4}+\lambda_{5}$ and $M^{2}$ $\equiv m_{3}^{2} / \sin \beta \cos \beta$. The vacuum expectation value $v(\sim 246$ $\mathrm{GeV}$ ) is equal to $\sqrt{2} \sqrt{\left\langle\phi_{1}^{0}\right\rangle^{2}+\left\langle\phi_{2}^{0}\right\rangle^{2}}$. Mass eigenstates for the $\mathrm{CP}$-even and the charged Higgs bosons are obtained by diagonalizing the mass matrices (7) and (9), respectively. The original Higgs boson fields $\phi_{1}, \phi_{2}, \omega^{-}$can be expressed in terms of the physical states and the Nambu-Goldstone modes $\left(G^{0}\right.$ and $\left.G^{ \pm}\right)$as

$$
\begin{aligned}
\phi_{1}^{0}= & \frac{1}{\sqrt{2}}\left[v \cos \beta+H \cos \alpha-h \sin \alpha+i\left(G^{0} \cos \beta\right.\right. \\
& -A \sin \beta)],
\end{aligned}
$$

\footnotetext{
${ }^{2}$ Our analyses will thus be valid in the cases where the effect of the bottom Yukawa coupling is sufficiently small; i.e., in the region of not too large $\tan \beta$.
}

where the angles $\alpha$ and $\chi$ are defined from the matrices that diagonalize the $2 \times 2$ matrices $M_{N}^{2}$ and $M_{S}^{2}$, respectively. That is, we have

$$
\begin{aligned}
& \left(\begin{array}{cc}
\cos (\alpha-\beta) & \sin (\alpha-\beta) \\
-\sin (\alpha-\beta) & \cos (\alpha-\beta)
\end{array}\right) \\
& \quad \times M_{N}^{2}\left(\begin{array}{cc}
\cos (\alpha-\beta) & -\sin (\alpha-\beta) \\
\sin (\alpha-\beta) & \cos (\alpha-\beta)
\end{array}\right)=\left(\begin{array}{cc}
m_{H}^{2} & 0 \\
0 & m_{h}^{2}
\end{array}\right) \\
& \left(\begin{array}{cc}
\cos \chi & \sin \chi \\
-\sin \chi & \cos \chi
\end{array}\right) M_{S}^{2}\left(\begin{array}{cc}
\cos \chi & -\sin \chi \\
\sin \chi & \cos \chi
\end{array}\right)=\left(\begin{array}{cc}
m_{S_{1}}^{2} & 0 \\
0 & m_{S_{2}}^{2}
\end{array}\right)
\end{aligned}
$$

where $m_{H}^{2}>m_{h}^{2}$ and $m_{S_{1}}^{2}>m_{S_{2}}^{2}$. The mixing angles $\alpha$ and $\chi$ then satisfy 


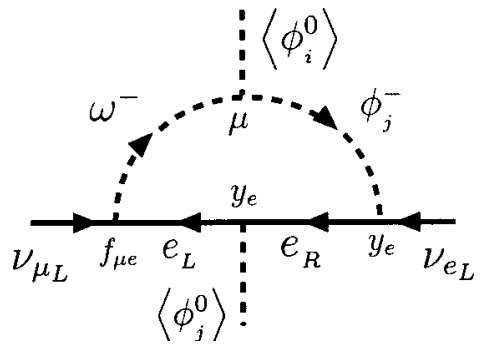

FIG. 1. A representative diagram that generates the neutrino mass. For type I, $i=1, j=2$, and for type II, $i=2, j=1$.

$$
\tan 2 \alpha=\frac{M^{2}-\left(\lambda_{3}+\lambda_{4}+\lambda_{5}\right) v^{2}}{M^{2}-\left(\lambda_{1} \cos ^{2} \beta-\lambda_{2} \sin ^{2} \beta\right) v^{2} / \cos 2 \beta} \tan 2 \beta,
$$

$\tan 2 \chi$

$$
=\frac{-\sqrt{2} \mu v}{M^{2}-m_{0}^{2}-\left(\lambda_{4}+\lambda_{5}+\sigma_{1} \cos ^{2} \beta+\sigma_{2} \sin ^{2} \beta\right) v^{2} / 2},
$$

which show that $\alpha$ and $\chi$ approach $\beta-\pi / 2$ and zero, respectively, ${ }^{3}$ when $M^{2}$ is much greater than $v^{2}, \mu^{2}$, and $m_{0}^{2}$; i.e., in the decoupling regime. In this limit, the massive Higgs bosons from the extra weak doublet are very heavy due to the large $M$ so that they are decoupled from the low energy observable.

Although neutrinos in this model are massless at the tree level, the loop diagrams involving charged Higgs bosons, as shown in Fig. 1, can generate Majorana mass terms for all three flavors of neutrinos. It was shown [2] that at the oneloop order the neutrino mass matrix, defined in the basis where the charged lepton Yukawa coupling constants are di- agonal in the lepton flavor space, is real and symmetric with vanishing diagonal elements. More explicitly, we have

$$
M_{\nu}=\left(\begin{array}{ccc}
0 & m_{12} & m_{13} \\
m_{12} & 0 & m_{23} \\
m_{13} & m_{23} & 0
\end{array}\right),
$$

with

$$
m_{i j}=f_{i j}\left(m_{e_{j}}^{2}-m_{e_{i}}^{2}\right) \mu \cot \beta \frac{1}{16 \pi^{2}} \frac{1}{m_{S_{1}}^{2}-m_{S_{2}}^{2}} \ln \frac{m_{S_{1}}^{2}}{m_{S_{2}}^{2}},
$$

where $m_{e_{i}}(i=1,2,3)$ is the charged lepton mass for type I. For type II, $\cot \beta$ should be replaced by $\tan \beta$. Note that Eq. (20) is valid for $m_{S_{i}} \gg m_{e_{j}}$.

The phenomenological analysis of the above mass matrix was given in Refs. [4], [5]. It was concluded that, in the Zee model, the bimaximal mixing solution is the only possibility to reconcile the atmospheric and solar neutrino data. Here we give a brief summary of these results, for completeness. Let us denote the three eigenvalues for the neutrino mass matrix [cf. Eq. (19)] as $m_{\nu_{1}}, m_{\nu_{2}}$, and $m_{\nu_{3}}$, which satisfy $m_{\nu_{1}}$ $+m_{\nu_{2}}+m_{\nu_{3}}=0$. The possible pattern of the neutrino mass spectrum allowed in the Zee model is $\left|m_{\nu_{1}}\right| \simeq\left|m_{\nu_{2}}\right| \gg\left|m_{\nu_{3}}\right|$, with $\quad m_{\nu_{1}}^{2}-m_{\nu_{3}}^{2} \simeq m_{\nu_{2}}^{2}-m_{\nu_{3}}^{2}=\Delta m_{\mathrm{atm}}^{2}, \quad$ and $\quad\left|m_{\nu_{1}}^{2}-m_{\nu_{2}}^{2}\right|$ $=\Delta m_{\text {solar }}^{2}$, where $\Delta m_{\mathrm{atm}}^{2}=O\left(10^{-3}\right) \mathrm{eV}^{2}$ from the atmospheric neutrino data, and $\Delta m_{\text {solar }}^{2}=O\left(10^{-5}\right) \mathrm{eV}^{2} \quad[\mathrm{M}$. Kheyer-Smirnov-Wolfenstein (MSW) large angle solution] or $O\left(10^{-10}\right) \mathrm{eV}^{2}$ (vacuum oscillation solution) from the solar neutrino data. ${ }^{4}$ Thus, we have $\left|m_{\nu_{1}}\right| \simeq\left|m_{\nu_{2}}\right| \simeq \sqrt{\Delta m_{\text {atm }}^{2}}\left(m_{\nu_{1}}\right.$ $\left.\simeq-m_{\nu_{2}}\right)$ and $\left|m_{\nu_{3}}\right| \simeq \Delta m_{\text {solar }}^{2} / 2 \sqrt{\Delta m_{\mathrm{atm}}^{2}}$. The approximate form of the neutrino mass matrix is given by

$$
M_{\nu}=\left(\begin{array}{ccc}
0 & \pm \sqrt{\left|m_{\nu_{1}} m_{\nu_{2}}\right| / 2} & \mp \sqrt{\left|m_{\nu_{1}} m_{\nu_{2}}\right| / 2} \\
\pm \sqrt{\left|m_{\nu_{1}} m_{\nu_{2}}\right| / 2} & 0 & -m_{\nu_{1}}-m_{\nu_{2}} \\
\mp \sqrt{\left|m_{\nu_{1}} m_{\nu_{2}}\right| / 2} & -m_{\nu_{1}}-m_{\nu_{2}} & 0
\end{array}\right),
$$

where the upper (lower) sign corresponds to $m_{\nu_{1}}<0(>0)$ case, and the corresponding Maki-Nakagawa-Sakata (MNS) matrix [18], which diagonalizes the neutrino mass matrix, is

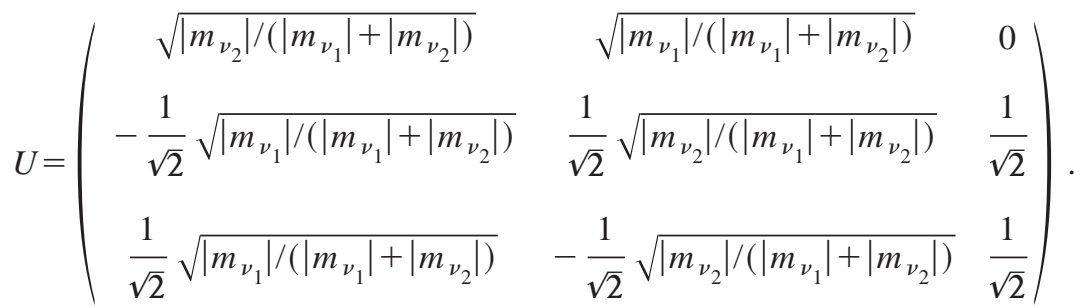

\footnotetext{
${ }^{3}$ Recall that we assumed $m_{H}>m_{h}$.

${ }^{4}$ Because of the structure of the mass matrix [cf. Eq. (19)] only the hierarchy pattern $\left|m_{\nu_{1}}\right| \simeq\left|m_{\nu_{2}}\right| \gg\left|m_{\nu_{3}}\right|$, rather than $\left|m_{\nu_{1}}\right| \simeq\left|m_{\nu_{2}}\right|$ $\ll\left|m_{\nu_{3}}\right|$, is realized in the Zee model [4,5].
} 
In the above equations, we took the limiting case where $U_{13}=0$ and $U_{32}=U_{23}=1 / \sqrt{2}{ }^{5}$ From Eqs. (20) and (21), we obtain

$$
\begin{aligned}
\left|\frac{f_{12}}{f_{13}}\right| & \simeq \frac{m_{\tau}^{2}}{m_{\mu}^{2}} \simeq 3 \times 10^{2}, \\
\left|\frac{f_{13}}{f_{23}}\right| & \simeq \frac{\sqrt{2} \Delta m_{\text {atm }}^{2}}{\Delta m_{\text {solar }}^{2}} \\
& \simeq \begin{cases}10^{2} & \text { (for the MSW large angle solution) }, \\
10^{7} & \text { (for the vacuum oscillation solution) } .\end{cases}
\end{aligned}
$$

Therefore, the magnitudes of the three coupling constants should satisfy the relation $\left|f_{12}\right| \gg\left|f_{13}\right| \gg\left|f_{23}\right|$. This hierarchy among the couplings $f_{i j}$ is crucial for our later discussion of the phenomenology of the singlet charged Higgs bosons.

For given values of the parameters $m_{S_{1}}, m_{S_{2}}, \tan \beta$, and $\mu$, the coupling constants $f_{i j}$ can be calculated from Eq. (20). For example, for $m_{S_{1}}=500 \mathrm{GeV}, m_{S_{2}}=100 \mathrm{GeV}, \tan \beta=1$, $\mu=100 \mathrm{GeV}$, and $m_{12}=3 \times 10^{-2} \mathrm{eV}$, we obtain $\left|f_{12}\right|$ $\sim 3 \times 10^{-4}$. In this example, when $S_{1}^{-}$is rather heavy and the lighter charged Higgs boson $S_{2}^{-}$is almost a weak singlet, i.e., the mixing angle $\chi$ approaches zero, it is unlikely that there are observable effects in the low energy data [7]; e.g., on the muon lifetime, the universality of tau decay into electrons or muons the rare decay of $\mu \rightarrow e \gamma$, the universality of $W$ boson decay into electrons, muons, or taus, and the decay width of $Z$ bosons. When $\left|f_{i j}\right|$ are small, we do not expect a large rate in the lepton flavor violation decay of a light neutral Higgs boson, such as $h \rightarrow \mu^{ \pm} e^{\mp}$ (the largest one), $h$ $\rightarrow e^{ \pm} \tau^{\mp}$, or $h \rightarrow \mu^{ \pm} \tau^{\mp}$ (the smallest one). On the contrary, as we will discuss in Sec. IV, the decay width of $h \rightarrow \gamma \gamma$ can significantly deviate from the SM value.

Finally, the phenomenological constraints on $f_{12}$ were derived in Ref. [6]. From the consistency of the muon decay rate and electroweak precision test it was found that

$$
\frac{f_{12}^{2}}{\bar{M}^{2}}<7 \times 10^{-4} G_{F},
$$

where $G_{F}$ is the Fermi constant, and

$$
\frac{1}{\bar{M}^{2}}=\frac{\sin ^{2} \chi}{m_{S_{1}}^{2}}+\frac{\cos ^{2} \chi}{m_{S_{2}}^{2}}
$$

This means that the $f_{i j}$ cannot be $O(1)$ unless the charged Higgs boson masses are of the order of $10 \mathrm{TeV}$.

\footnotetext{
${ }^{5}$ This limit corresponds to $\theta_{2}=\pi / 4$ and $\theta_{3}=0$ in the notation of Ref. [18].
}

\section{HIGGS BOSON MASS AND COUPLINGS THROUGH RGE'S}

In this section, we determine the bounds on the mass of the lightest $C P$-even Higgs boson as a function of the cutoff scale of the Zee model by analyzing the set of renormalization group equations. We also study the allowed ranges of the coupling constants, especially $\sigma_{1}$ and $\sigma_{2}$ in Eq. (4). In Sec. $\mathrm{IV}$, they will be used to evaluate how much the partial decay width of $h \rightarrow \gamma \gamma$ can deviate from its SM value due to the one-loop contribution from the singlet charged Higgs boson.

The mass bounds are determined in the following manner. For each set of parameters defined at the electroweak scale, the running coupling constants are calculated numerically through RGE's at the one-loop level. We require that all the dimensionless coupling constants do not blow up below a given cutoff scale $\Lambda$, and the coupling constants satisfy the vacuum stability condition. We vary the input parameters at the electroweak scale and determine the possible range of the lightest $C P$-even Higgs boson mass as a function of $\Lambda$. In a similar manner, we also study the allowed ranges of various Higgs boson self-coupling constants at the electroweak scale and as a function of the lightest $C P$-even Higgs boson mass.

We derived the one-loop RGE's for the Zee model, and listed them in the Appendix for reference. For simplicity, in the RGE's, we neglected all the Yukawa coupling constants $\left(y_{u}, y_{d}, y_{e}\right)$ but the top Yukawa coupling $y_{t} \cdot{ }^{6}$ Although we kept the new coupling constants $f_{i j}$ in the RGE's listed in the Appendix, we neglected $f_{i j}$ in the numerical calculation. This is because the magnitudes of these coupling constants are numerically too small to affect the final results unless the singlet charged scalar boson mass is larger than a few $\mathrm{TeV}$ [cf. Eq. (25)]. The dimensionless coupling constants relevant to our numerical analysis are the three gauge coupling constants $g_{1}, g_{2}, g_{3}$, the top Yukawa coupling constant $y_{t}$, and eight scalar self-coupling constants $\lambda_{i}(i=1-5)$ and $\sigma_{i}(i$ $=1-3)$. There are five dimensionful parameters in the Higgs potential, namely, $m_{1}^{2}, m_{2}^{2}, m_{3}^{2}, m_{0}^{2}$, and $\mu$. Instead of $m_{1}^{2}, \quad m_{2}^{2}$, and $m_{3}^{2}$, we take $v, \tan \beta$, and $M^{2}$ $\equiv m_{3}^{2} / \sin \beta \cos \beta$ as independent parameters, where $v(\sim 246$ $\mathrm{GeV})$ characterizes the weak scale and $M$ the soft-breaking scale of the discrete symmetry.

In the actual numerical calculation we first fix $\tan \beta$ and $M$. For a given mass $\left(m_{h}\right)$ of the lightest $C P$-even Higgs boson, we solve one of the $\lambda_{i}$, which is chosen to be $\lambda_{3}$ here, in terms of the other $\lambda_{i}$. We then numerically evaluate all dimensionless coupling constants according to the RGE's. From $m_{h}$ to $M$ we use the SM RGE's, which are matched to the Zee model RGE's at the soft-breaking scale $M^{7}$

\footnotetext{
${ }^{6}$ In the model with type-II Yukawa interaction, the bottom quark Yukawa interaction can become important for large $\tan \beta$.

${ }^{7}$ The parameters $m_{0}$ and $\mu$ are relevant only to the charged scalar mass matrix. In principle, our numerical results also depend on these parameters through the renormalization of various coupling constants from the scale of $m_{h}$ to the charged scalar mass. Since these effects are expected to be small, we calculate the RGE's as if all the scalar bosons except $h$ decouple at the scale $M$.
} 
We require the following two conditions to be satisfied for each scale $Q$ up to a given cutoff scale $\Lambda$.

(1) Applicability of the perturbation theory implies

$$
\lambda_{i}(Q)<8 \pi, \quad \sigma_{i}(Q)<8 \pi, \quad y_{t}^{2}(Q)<4 \pi .
$$

(2) The vacuum stability conditions must be satisfied. The requirement that quartic coupling terms of the scalar potential do not have a negative coefficient in any direction leads to the following conditions at each renormalization scale $Q$ :

(a)

$$
\lambda_{l}(Q)>0, \quad \lambda_{2}(Q)>0, \quad \sigma_{3}(Q)>0,
$$

(b)

$$
\begin{gathered}
\sigma_{1}(Q)+\sqrt{\lambda_{1}(Q) \sigma_{3}(Q) / 2}>0, \\
\sigma_{2}(Q)+\sqrt{\lambda_{2}(Q) \sigma_{3}(Q) / 2}>0, \\
\bar{\lambda}(Q)+\sqrt{\lambda_{1}(Q) \sigma_{2}(Q)}>0,
\end{gathered}
$$

where

$\bar{\lambda}(Q)=\lambda_{3}(Q)+\min \left[0, \lambda_{4}(Q)+\lambda_{5}(Q), \lambda_{4}(Q)-\lambda_{5}(Q)\right]$.

(c) If $\sigma_{1}(Q)<0$ and $\sigma_{2}(Q)<0$, then

$$
\begin{aligned}
\bar{\lambda}(Q) & +\frac{2}{\sigma_{3}(Q)}\left\{\left[( \frac { \lambda _ { 1 } ( Q ) \sigma _ { 3 } ( Q ) } { 2 } - \sigma _ { 1 } ^ { 2 } ( Q ) ) \left(\frac{\lambda_{2}(Q) \sigma_{3}(Q)}{2}\right.\right.\right. \\
& \left.\left.\left.-\sigma_{2}^{2}(Q)\right)\right]^{1 / 2}-\sigma_{1}(Q) \sigma_{2}(Q)\right\}>0
\end{aligned}
$$

If $\sigma_{1}(Q)<0$ and $\bar{\lambda}(Q)<0$, then

$$
\begin{gathered}
\sigma_{2}(Q)+\frac{1}{\lambda_{1}(Q)}\left\{\left[[ \lambda _ { 1 } ( Q ) \lambda _ { 2 } ( Q ) - \overline { \lambda } ^ { 2 } ( Q ) ] \left(\frac{\lambda_{1}(Q) \sigma_{3}(Q)}{2}\right.\right.\right. \\
\left.\left.\left.-\sigma_{1}^{2}(Q)\right)\right]^{1 / 2}-\sigma_{1}(Q) \bar{\lambda}(Q)\right\}>0
\end{gathered}
$$

If $\sigma_{2}(Q)<0$ and $\bar{\lambda}(Q)<0$, then

$$
\begin{gathered}
\sigma_{1}(Q)+\frac{1}{\lambda_{2}(Q)}\left\{\left[[ \lambda _ { 1 } ( Q ) \lambda _ { 2 } ( Q ) - \overline { \lambda } ^ { 2 } ( Q ) ] \left(\frac{\lambda_{2}(Q) \sigma_{3}(Q)}{2}\right.\right.\right. \\
\left.\left.\left.-\sigma_{2}^{2}(Q)\right)\right]^{1 / 2}-\sigma_{2}(Q) \bar{\lambda}(Q)\right\}>0
\end{gathered}
$$

[When $\sigma_{1}(Q), \sigma_{2}(Q)$, and $\bar{\lambda}(Q)$ are all negative, the above three conditions are equivalent.]

In addition to the above conditions, we also demand local stability of the potential at the electroweak scale, namely, we calculate the mass spectrum of all scalar fields at the extremum of the potential and demand that all eigenvalues of the

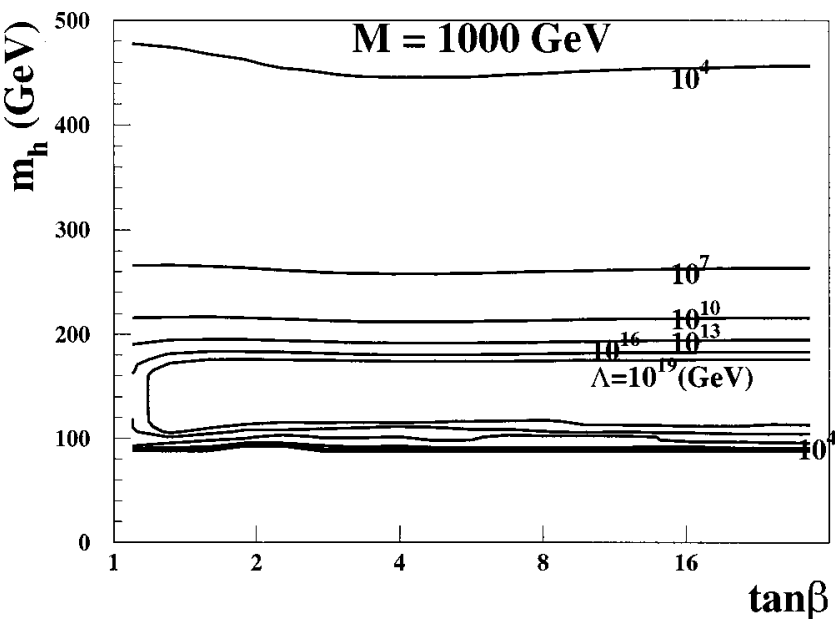

FIG. 2. The allowed mass range of the lightest $C P$-even Higgs boson for $M=1000 \mathrm{GeV}$. $\Lambda$ is the cutoff scale.

squared scalar mass are positive. We scan the remaining seven-dimensional space of $\lambda_{i}$ and $\sigma_{i}$ and examine whether a given mass of the lightest $C P$-even Higgs boson is allowed under the above conditions. In this way we obtain the allowed range of $m_{h}$ as a function of $\tan \beta$ and $M$, for each value of the cutoff scale $\Lambda$.

First, we discuss our result in the decoupling case, in which the soft-breaking scale $M$ is much larger than the electroweak scale $\sim v$, and the masses of all the Higgs bosons but $h$ (and $S_{2}$ ) are at the order of $M{ }^{8}$ In Fig. 2, the allowed range of $m_{h}$ is shown as a function of $\tan \beta$ for $M$ $=1000 \mathrm{GeV}$. [We take the pole mass of the top quark $m_{t}$ $=175 \mathrm{GeV}, \alpha_{s}\left(m_{Z}\right)=0.118$ for numerical calculation.] The allowed ranges are shown as contours for six different values of $\Lambda$, i.e., $\Lambda=10^{19}, 10^{16}, 10^{13}, 10^{10}, 10^{7}$, and $10^{4} \mathrm{GeV}$. For most values of $\tan \beta$, except for the small $\tan \beta$ region, the upper bound of $m_{h}$ is about $175 \mathrm{GeV}$ and the lower bound is between 110 and $120 \mathrm{GeV}$ for the cutoff scale $\Lambda$ to be near the Planck scale. The numerical values in this figure are very close to those in the corresponding figure for the THDM discussed in Ref. [13]. Compared to the corresponding lower mass bound in the $\mathrm{SM}$, which is $145 \mathrm{GeV}$ when using the one-loop RGE's, the lower mass bound in this model is reduced by about 30 to $40 \mathrm{GeV}$. The reason is similar to the THDM case: the lightest $C P$-even Higgs boson mass is essentially determined by the value of $\lambda_{2}$ for $\tan \beta$ to be larger

\footnotetext{
${ }^{8}$ In the decoupling regime $(M \rightarrow \infty$, which leads to $\alpha \rightarrow \beta-\pi / 2$ and $\chi \rightarrow 0$ ), the masses of $h$ and $S_{2}$ are dominated by the (11) component of the mass matrix in Eq. (7) and the (22) component of that in Eq. (9), respectively. The mass of $h$ is determined by the self-coupling constants $\lambda_{i}$, while that of $S_{2}$ depends not only on the self-couplings constants $\sigma_{i}$ but also on the free mass parameter $m_{0}$. As noted in footnote 7, from $m_{h}$ to $M$, the SM RGE's are used in our analysis, even if the mass of $S_{2}$ is smaller than $M$. The effect of $S_{2}$ on the mass bound of $h$ is expected to be small, because at the one-loop level the primary effect is through the running of $g_{1}$, whose contribution to the right-handed side of the RGE for the Higgs self-coupling constant is small.
} 


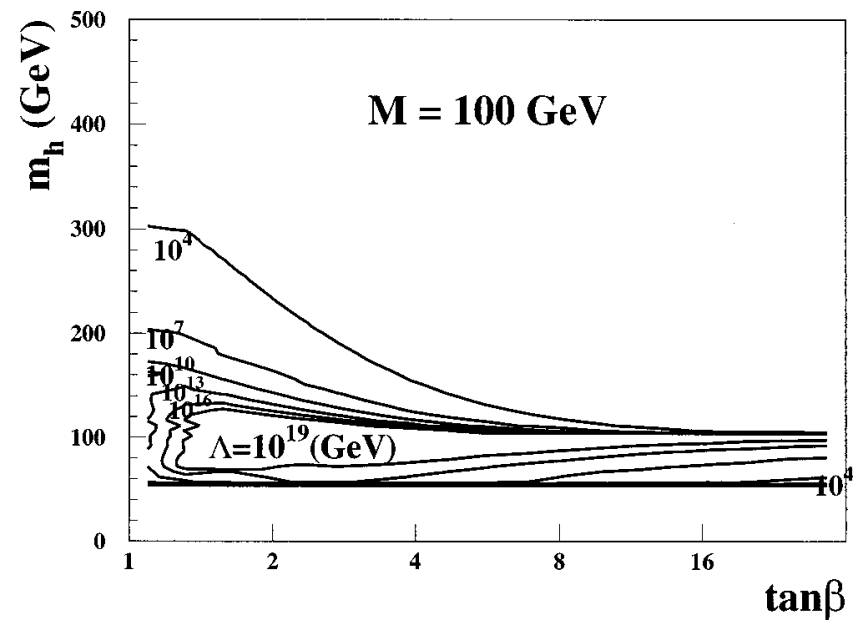

FIG. 3. The allowed mass range of the lightest $C P$-even Higgs boson for $M=100 \mathrm{GeV}$.

than about 2, where $\lambda_{2}$ plays the role of the self-coupling constant of the Higgs potential in the SM. ${ }^{9}$ On the right-hand side of the RGE for $\lambda_{2}$ [cf. Eq. (A5)] there are additional positive-definite terms $\left(2 / 16 \pi^{2}\right)\left[\lambda_{3}^{2}+\left(\lambda_{3}+\lambda_{4}\right)^{2}+\lambda_{5}^{2}+\sigma_{2}^{2}\right]$ as compared to the RGE for the Higgs self-coupling constant in the SM. These additional terms can improve vacuum stability, and allow lower values of $m_{h}$. Therefore, one of the features of the model is to have a different mass range for the lightest $C P$-even Higgs boson as compared to the SM Higgs boson, for a given cutoff scale.

Next, we show our result for $M$ to be around $v$. In Fig. 3, we present the $m_{h}$ bound for $M=100 \mathrm{GeV}$. In this case, the allowed range of $m_{h}$ is reduced as compared to that in the decoupling case, and lies around $m_{h} \sim M$ for large $\tan \beta$. Notice that we have not included phenomenological constraints from the $b \rightarrow s \gamma, \rho$ parameter, and direct Higgs boson search experiment at LEP. As mentioned before, the mass bounds obtained from the RGE analysis are the same for the type-I and type-II models without these phenomenological constraints. However, it was shown in Ref. [13] that the $b$ $\rightarrow s \gamma$ data can put a strong constraint on the allowed range of the Higgs boson mass for $M \lessgtr 200-400 \mathrm{GeV}$ in the type-II THDM, whereas there is no appreciable effect in the type-I model. This is because a small $M$ implies a light charged Higgs boson in the THDM, which can induce a large decay branching ratio for $b \rightarrow s \gamma$ in the type-II model [19]. ${ }^{10} \mathrm{We}$ expect a similar constraint from the $b \rightarrow s \gamma$ data on the type-II Zee model, when $M$ is small.

In Fig. 4, we show the upper and lower bounds of $m_{h}$ as a function of $M$ for various values of $\Lambda$. For given $M$, we scan the range of $\tan \beta$ for $1 \leqslant \tan \beta \leqslant 16 \sqrt{2}(\simeq 22.6)$. We find that the $m_{h}$ bounds obtained are almost the same as those for the THDM. The primary reason for this is that the new cou-

\footnotetext{
${ }^{9}$ However, $\tan \beta$ cannot be too large to ignore the contribution of the bottom quark in the case with type-II Yukawa interaction.

${ }^{10}$ In addition, it is known that the $R_{b}$ data also give strong constraints on the charged Higgs bosons in the type-II THDM [20].
}

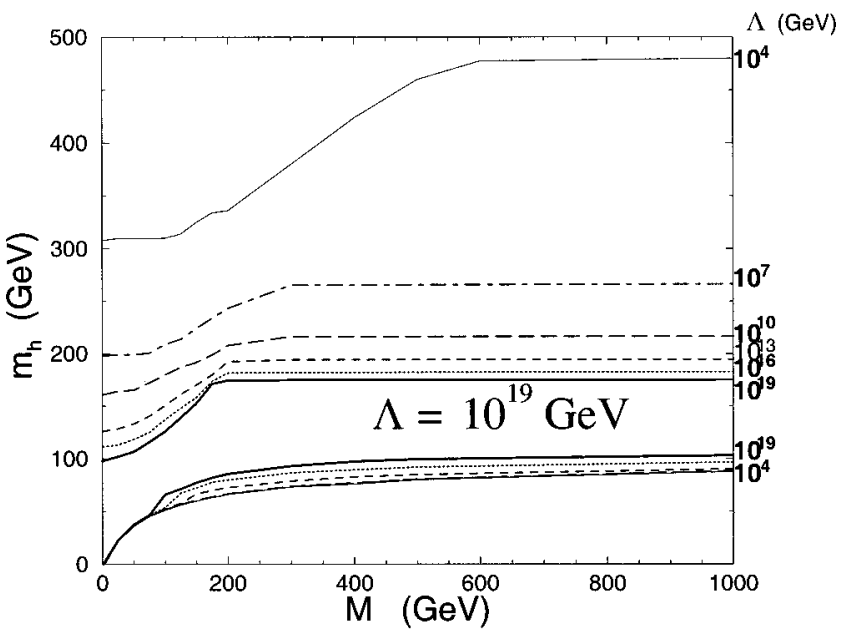

FIG. 4. The allowed ranges of the lightest $C P$-even Higgs boson mass as a function of $M$ for various $\Lambda$ values.

pling constants $\sigma_{1}, \sigma_{2}$, and $\sigma_{3}$ do not appear directly in the mass formula for $m_{h}$, and therefore do not induce large effects on the bounds of $m_{h}$.

We also investigate the allowed range of coupling constants $\sigma_{1}, \sigma_{2}$, and $\sigma_{3}$. For this purpose, we fix $\sigma_{1}$ (or $\left.\sigma_{2}, \sigma_{3}\right)$ as well as $\tan \beta$ and $M$ to evaluate the upper and lower bounds of $m_{h}$ for each $\Lambda$ value. In this way, we determine the possible range of $\sigma_{1}$ (or $\sigma_{2}, \sigma_{3}$ ) under the condition that the theory does not break down below the cutoff scale $\Lambda$. In Fig. 5, we present the allowed range of $\sigma_{1}$ and $m_{h}$ for different choices of $\Lambda$ in the case of $M=1000 \mathrm{GeV}$ and $\tan \beta=\sqrt{2}$ or $16 \sqrt{2}$. A similar figure is shown for the possible range of $\sigma_{2}$ in Fig. 6 . We see that the maximal value of $\sigma_{1}$ and $\sigma_{2}$ is around 0.7 for $m_{h}=110-170 \mathrm{GeV}$ if we take the cutoff scale to be $10^{19} \mathrm{GeV}$. For smaller values of $\Lambda$ the allowed range of $\sigma_{i}$ becomes larger. For example, $\sigma_{1}$ can exceed one for $\Lambda=10^{13} \mathrm{GeV}$. We have calculated the results for other value of $\tan \beta$ and checked that these figures do not change greatly between $\tan \beta=1.4$ and $16 \sqrt{2}$. We also present the allowed range in the $\sigma_{1}$ and $\sigma_{2}$ plane for a fixed value of $m_{h}$ in Figs. 7 and 8 for $m_{h}=125$ and $140 \mathrm{GeV}$, respectively. For either value of $m_{h}$ with $\tan \beta=16 \sqrt{2}$, both $\sigma_{1}$ and $\sigma_{2}$ can be as large as 0.5 (2) for $\Lambda=10^{19}\left(10^{7}\right) \mathrm{GeV}$. The allowed range of $\sigma_{3}$ and $m_{h}$ for various values of $\Lambda$ is given in Fig. 9. It is shown that $\sigma_{3}$ has to be larger than zero, due to the vacuum stability condition. The maximal value of $\sigma_{3}$ is about 1 (3) for $\Lambda=10^{19}\left(10^{7}\right) \mathrm{GeV}$ and $M=1000 \mathrm{GeV}$. The impact of these new coupling constants on collider phenomenology is discussed in the next section.

\section{TWO-PHOTON DECAY WIDTH OF THE NEUTRAL HIGGS BOSON}

In this section, we study the phenomenological consequences of the Higgs boson mass and the Higgs boson coupling constants derived in the previous section. The important feature of the Higgs sector of the Zee model is that there are an additional weak doublet and a singlet charged Higgs boson. The physical states of the Higgs particles are two 


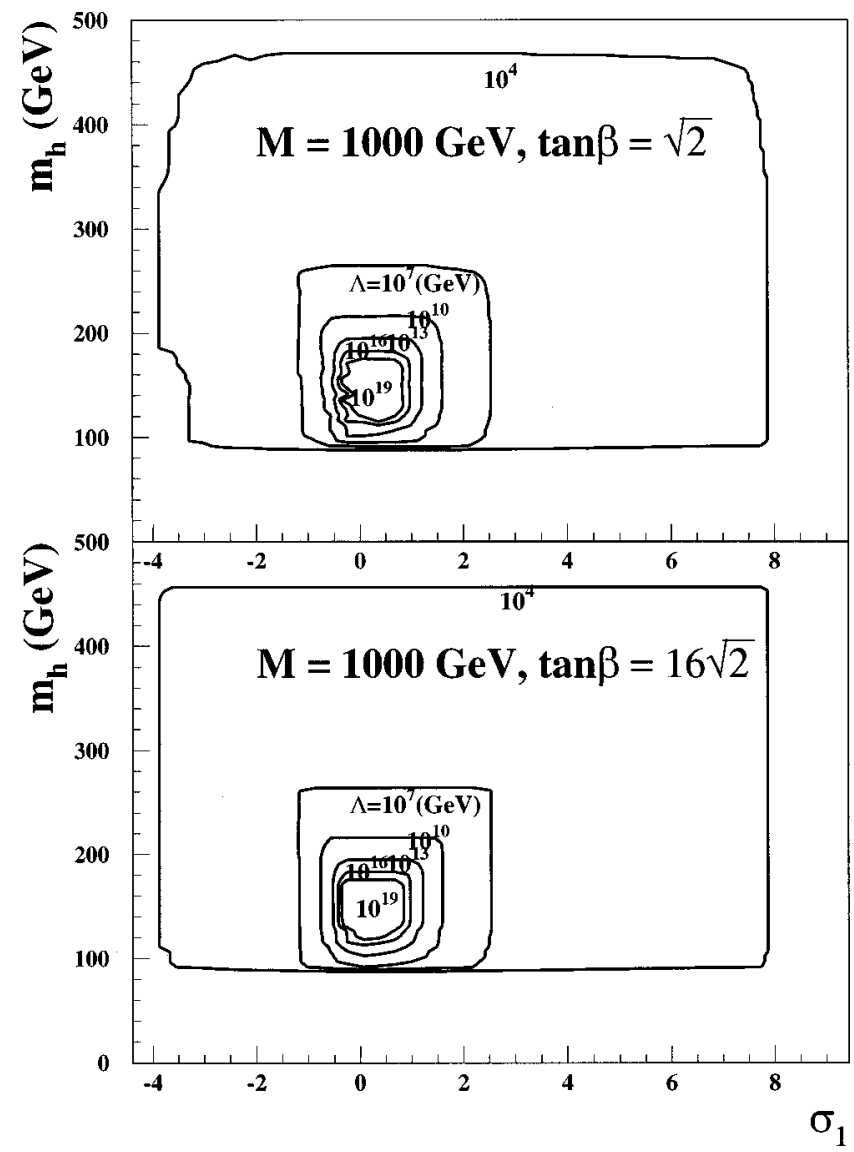

FIG. 5. The allowed range of $\sigma_{1}$ and $m_{h}$ for various $\Lambda$ values.

$C P$-even Higgs bosons, one $C P$-odd Higgs boson, and two pairs of charged Higgs bosons. Therefore, the Higgs phenomenology is quite close to that in the ordinary two-Higgsdoublet model. One unique difference is the existence of the additional weak-singlet charged Higgs boson. The effect of this extra charged Higgs boson is especially important when $M$ is much larger than the $Z$ boson mass, i.e., in the decoupling regime. In such a case, the heavier $C P$-even Higgs boson, the $C P$-odd Higgs boson, as well as one of the charged Higgs bosons have masses approximately equal to $M$, and these heavy states are decoupled from low energy observables. (Note that the condition on the applicability of perturbation theory forbids too large self-couplings among the Higgs bosons. Hence, in the limit of large $M$, the heavy Higgs bosons decouple from the low energy effective theory.) The remaining light states are the lighter $C P$-even Higgs boson $h$ and the lighter charged Higgs boson $S_{2}$ which mainly comes from the weak singlet. In the previous section, we showed that, even in the decoupling case, there can be large differences in the allowed range of $m_{h}$ between the Zee model and the SM. Similarly, we expect that, even in the decoupling case, the presence of the additional weak-singlet charged Higgs boson can give rise to interesting Higgs phenomenology.

Since the lighter charged Higgs boson $S_{2}$ can couple to Higgs bosons and leptons, it can affect the decay and production of neutral Higgs bosons at colliders through radiative corrections. In the following, we consider the decay width of

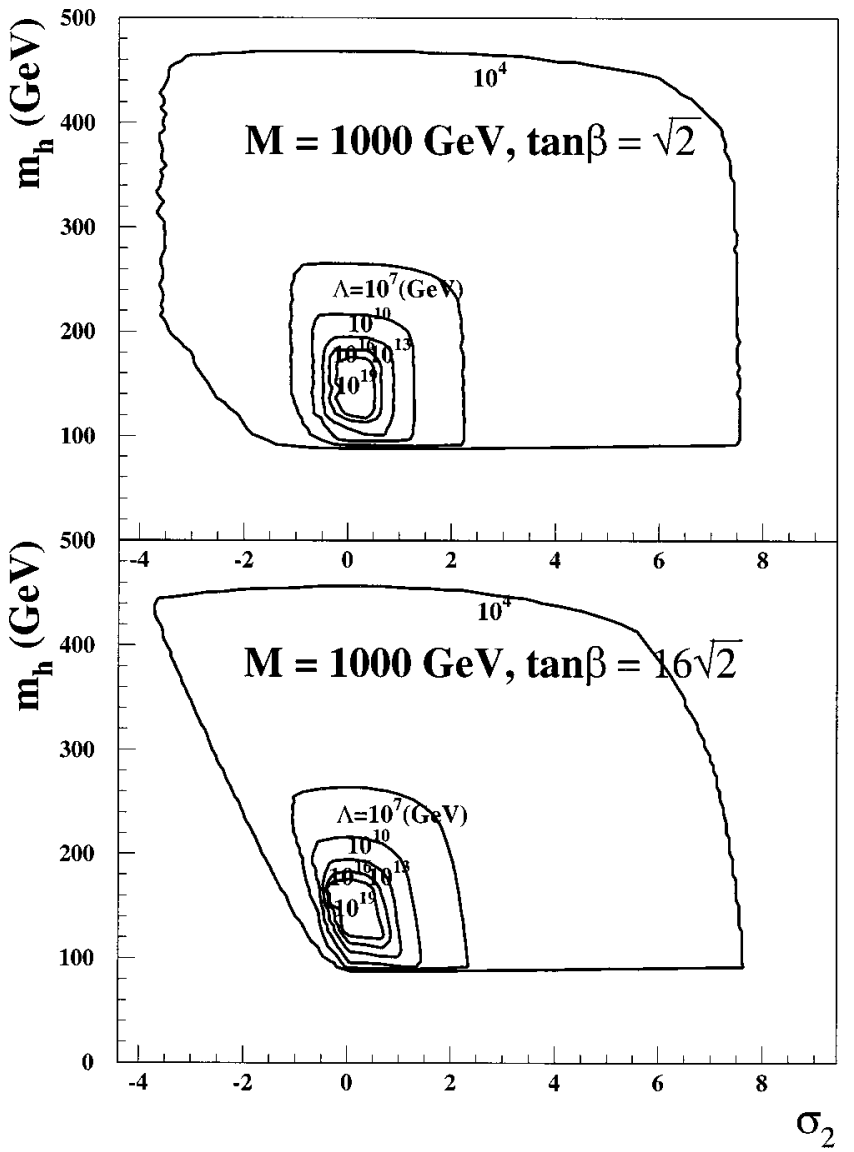

FIG. 6. The allowed range of $\sigma_{2}$ and $m_{h}$ for various $\Lambda$ values.

$h \rightarrow \gamma \gamma$ as an example. For a SM Higgs boson, the partial decay width (or branching ratio) of $h \rightarrow \gamma \gamma$ is small: $\sim 9.2$ $\mathrm{keV}$ (or $2.2 \times 10^{-3}$ ) for $m_{h}=125 \mathrm{GeV}$, and $\sim 15.4 \mathrm{keV}$ (or $1.9 \times 10^{-3}$ ) for $m_{h}=140 \mathrm{GeV}$, with a $175 \mathrm{GeV}$ top quark. Nevertheless, it is an important discovery mode of the Higgs boson at the LHC experiments for $m_{h}$ less than twice the $W$ boson mass. Needless to say, a change in the branching ratio of $h \rightarrow \gamma \gamma$ would lead to a different production rate of $p p$ $\rightarrow h X \rightarrow \gamma \gamma X$. At future $e^{+} e^{-}$LC's, the branching ratio of $h \rightarrow \gamma \gamma$ can be determined via the reaction $e^{+} e^{-} \rightarrow q \bar{q} \gamma \gamma$ and $e^{+} e^{-} \rightarrow \nu \bar{\nu} \gamma \gamma$ with 16-22\% accuracy [16]. At the photon-photon collision option of future LC's, the partial decay width of $h \rightarrow \gamma \gamma$ can be precisely tested within $2 \%$ accuracy [17] by measuring the inclusive production rate of the Higgs boson $h$. Clearly, a change in the partial decay width of $h \rightarrow \gamma \gamma$ will lead to a different production rate for $h$. In the Zee model, such a change is expected after taking into account the loop contribution of the extra charged Higgs boson. We find that the deviation from the SM prediction can be sizable and therefore testable at the LHC and future LC's.

The partial decay width of $h \rightarrow \gamma \gamma$ is calculated at the one-loop order. As in our previous discussion, we limit ourselves to the parameter space in which $1 \leqslant \tan \beta \leqslant 16 \sqrt{2}$, and keep only the top quark contribution from the fermionic loop diagrams. Including the loop contributions from the $W$ boson and the charged Higgs bosons $S_{1}$ and $S_{2}$ together with the top quark loop contribution, we obtain [21] 


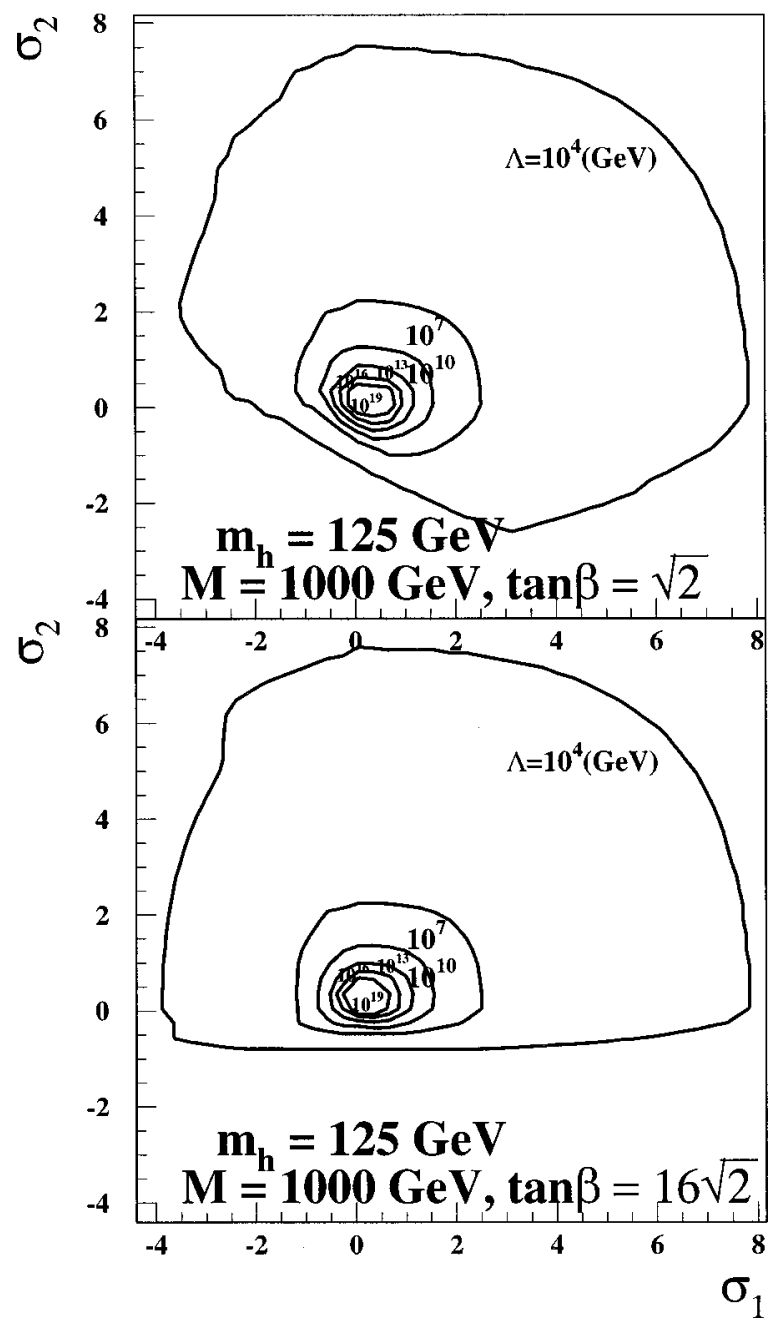

FIG. 7. The allowed range of $\sigma_{1}$ and $\sigma_{2}$ for $m_{h}=125 \mathrm{GeV}$.

$$
\Gamma(h \rightarrow \gamma \gamma)=\frac{\left(\alpha m_{h}\right)^{3}}{256 \pi^{2} \sin ^{2} \theta_{W} m_{W}^{2}}\left|\sum_{i=S_{1}, S_{2}, t, W} I_{i}\right|^{2},
$$

with

$$
\begin{aligned}
I_{S_{1}} & =R_{S_{1}} F_{0}\left(r_{i}\right), \\
I_{S_{2}} & =R_{S_{2}} F_{0}\left(r_{i}\right), \\
I_{t} & =\frac{4}{3}\left(\frac{\cos \alpha}{\sin \beta}\right) F_{1 / 2}\left(r_{i}\right), \\
I_{W} & =\sin (\beta-\alpha) F_{1}\left(r_{i}\right),
\end{aligned}
$$

where $r_{i}=4 m_{i}^{2} / m_{h}^{2}$ and $m_{i}$ is the mass of the internal lines in the loop diagram. $R_{S_{1}}$ and $R_{S_{2}}$ are given by

$$
\begin{aligned}
R_{S_{1}}= & \frac{v^{2}}{2} \frac{1}{m_{S_{1}}^{2}}\left[\operatorname { c o s } ^ { 2 } \chi \left\{-\lambda_{1} \sin \alpha \sin ^{2} \beta \cos \beta\right.\right. \\
& +\lambda_{2} \cos \alpha \sin \beta \cos ^{2} \beta+\lambda_{3}\left(\cos \alpha \sin ^{3} \beta\right. \\
& \left.\left.-\sin \alpha \cos ^{3} \beta\right)-\frac{1}{2}\left(\lambda_{4}+\lambda_{5}\right) \cos (\alpha+\beta) \sin 2 \beta\right\}
\end{aligned}
$$

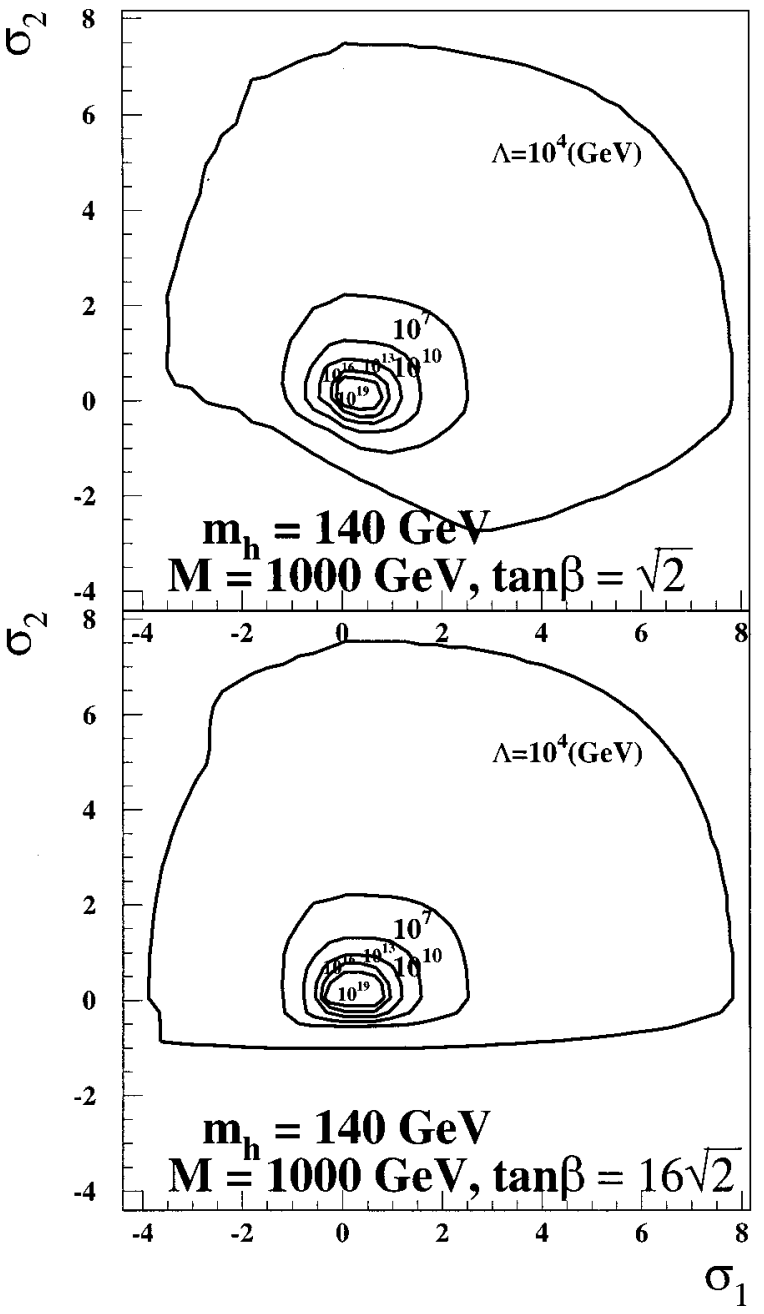

FIG. 8. The allowed range of $\sigma_{1}$ and $\sigma_{2}$ for $m_{h}=140 \mathrm{GeV}$.

$$
\begin{aligned}
& +\sin ^{2} \chi\left\{-\sigma_{1} \sin \alpha \cos \beta+\sigma_{2} \cos \alpha \sin \beta\right\} \\
& \left.+\sqrt{2} \sin \chi \cos \chi \frac{\mu}{v} \sin (\alpha-\beta)\right],
\end{aligned}
$$

$$
\begin{aligned}
R_{S_{2}}= & \frac{v^{2}}{2} \frac{1}{m_{S_{2}}^{2}}\left[\operatorname { s i n } ^ { 2 } \chi \left\{-\lambda_{1} \sin \alpha \sin ^{2} \beta \cos \beta\right.\right. \\
& +\lambda_{2} \cos \alpha \sin \beta \cos ^{2} \beta+\lambda_{3}\left(\cos \alpha \sin ^{3} \beta\right. \\
& \left.\left.-\sin \alpha \cos ^{3} \beta\right)-\frac{1}{2}\left(\lambda_{4}+\lambda_{5}\right) \cos (\alpha+\beta) \sin 2 \beta\right\} \\
& +\cos ^{2} \chi\left\{-\sigma_{1} \sin \alpha \cos \beta+\sigma_{2} \cos \alpha \sin \beta\right\} \\
& \left.-\sqrt{2} \sin \chi \cos \chi \frac{\mu}{v} \sin (\alpha-\beta)\right],
\end{aligned}
$$

and

$$
\begin{aligned}
F_{0}(r) & =r[1-r f(r)], \\
F_{1 / 2}(r) & =-2 r[1+(1-r) f(r)], \\
F_{1}(r) & =2+3 r+3 r(2-r) f(r),
\end{aligned}
$$




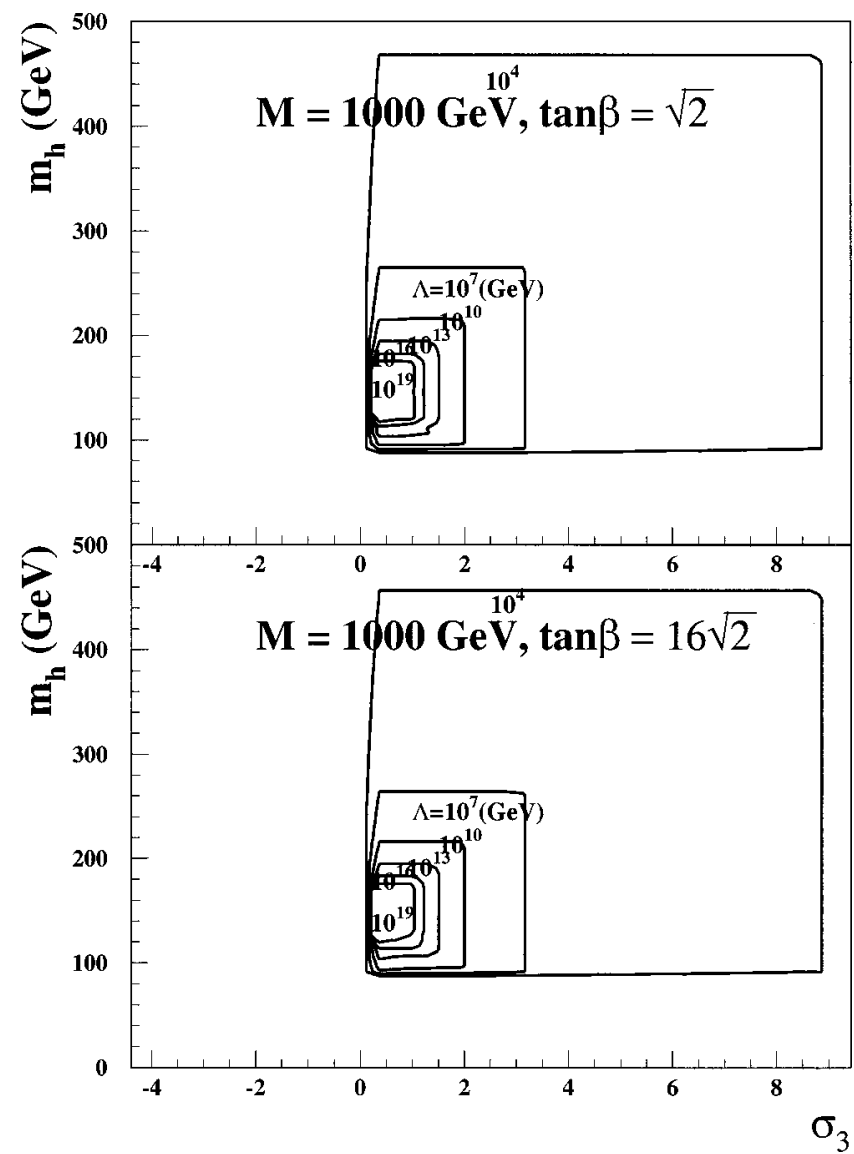

FIG. 9. The allowed range of $\sigma_{3}$ and $m_{h}$ for various $\Lambda$ values.

with

$$
f(r)=\left\{\begin{array}{cc}
{\left[\sin ^{-1}(\sqrt{1 / r})\right]^{2}} & \text { if } r \geqslant 1, \\
-\frac{1}{4}\left[\ln \frac{1+\sqrt{1-r}}{1-\sqrt{1-r}}-i \pi\right]^{2} & \text { if } r<1 .
\end{array}\right.
$$

In the decoupling case of the model, namely, $M^{2} \gg \lambda_{i} v^{2}$, the above formulas are greatly simplified. This limit corresponds to $\alpha \rightarrow \beta-\pi / 2$ and $\chi \rightarrow 0$, so that the light charged Higgs boson $S_{2}^{ \pm}$is identical to the weak-singlet Higgs boson $\omega^{ \pm}$. Thus, we have

$$
R_{S_{2}} \rightarrow \frac{v^{2}}{2} \frac{1}{m_{S_{2}}^{2}}\left(\sigma_{1} \cos ^{2} \beta+\sigma_{2} \sin ^{2} \beta\right),
$$

and both the top quark and the $W$ boson loop contributions are reduced to their SM values. We wish to stress that the weak-singlet Higgs boson does not directly couple to the quark fields in the limit of $\chi \rightarrow 0$. Therefore, it does not affect the decay rate of $b \rightarrow s \gamma$ at one-loop order. Similarly, being a weak singlet, it also gives no contribution to the $\rho$ parameter. Hence the low energy constraint from either the $b \rightarrow s \gamma$ decay or the $\rho$ parameter on the Zee model in the limit of $\chi$ $\rightarrow 0$ is similar to their effects on the THDM. Let us examine at the one-loop level the effect of the weak-singlet charged Higgs boson on the decay width of $h \rightarrow \gamma \gamma$ in the decoupling limit. Let us recall that in Fig. 8 the size of the new couplings $\sigma_{1}$ and $\sigma_{2}$ can be as large as 2 simultaneously, if the cutoff scale is of the order of $10^{7} \mathrm{GeV}$. For the Zee model to be a valid low energy effective theory up to $10^{19} \mathrm{GeV}, \sigma_{1}$ and $\sigma_{2}$ cannot be much larger than 0.6. To illustrate the implications of this result, we show in Figs. 10(a) and 10(b) the ratio $(r)$ of the $h \rightarrow \gamma \gamma$ width predicted in the Zee model to that in the $\mathrm{SM}, r \equiv \Gamma_{\mathrm{Zee}}(h \rightarrow \gamma \gamma) / \Gamma_{\mathrm{SM}}(h \rightarrow \gamma \gamma)$, as a function of the coupling constant $\sigma_{2}$ and the charged Higgs boson mass $m_{S_{2}}$. Here, for simplicity, we have set $\sigma_{1}=\sigma_{2}$ so that the $\tan \beta$ dependence drops out in the decoupling case [cf. Eq. (42)]. For illustration, we consider two cases for the mass of the lighter $C P$-even Higgs boson: $m_{h}=125$ and $140 \mathrm{GeV}$. As shown in the figures, the ratio $r$ can be around 0.8 for $\sigma_{1}=\sigma_{2} \equiv \sigma \approx 0.5$ and $m_{S_{2}} \approx 100 \mathrm{GeV}$. This reduction is due to the cancellation between the contribution from the $S_{2}$ boson loop and the $W$ boson loop contributions. To have a

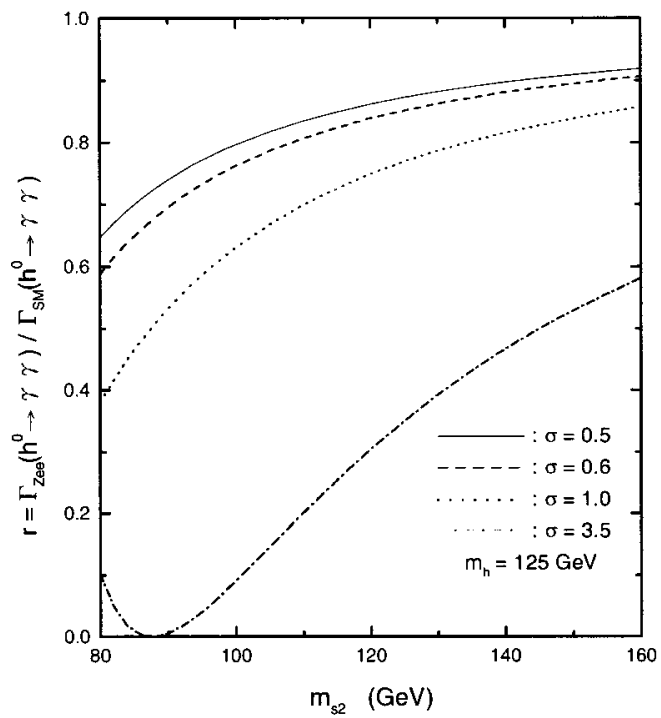

(a)

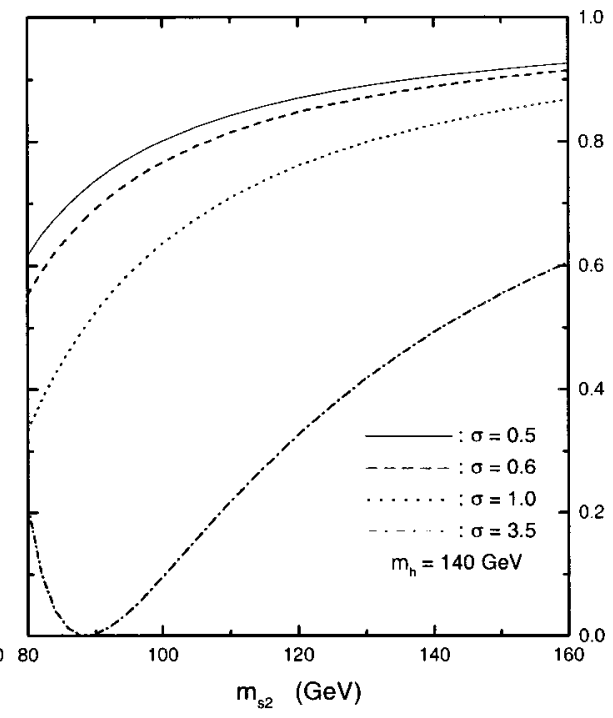

(b)
FIG. 10. (a) The ratio $r$ as a function of the charged Higgs boson mass $m_{S_{2}}$ for various values of the coupling constants $\sigma_{1}=\sigma_{2}$ $\equiv \sigma$ with $m_{h}=125 \mathrm{GeV}$. The two smaller $\sigma$ 's are consistent with the cutoff scales $\Lambda=10^{19}$ and $10^{16} \mathrm{GeV}$, respectively. The two larger $\sigma$ 's are allowed for $\Lambda$ $=10^{4} \mathrm{GeV}$. (b) A similar plot with $m_{h}=140 \mathrm{GeV}$. 


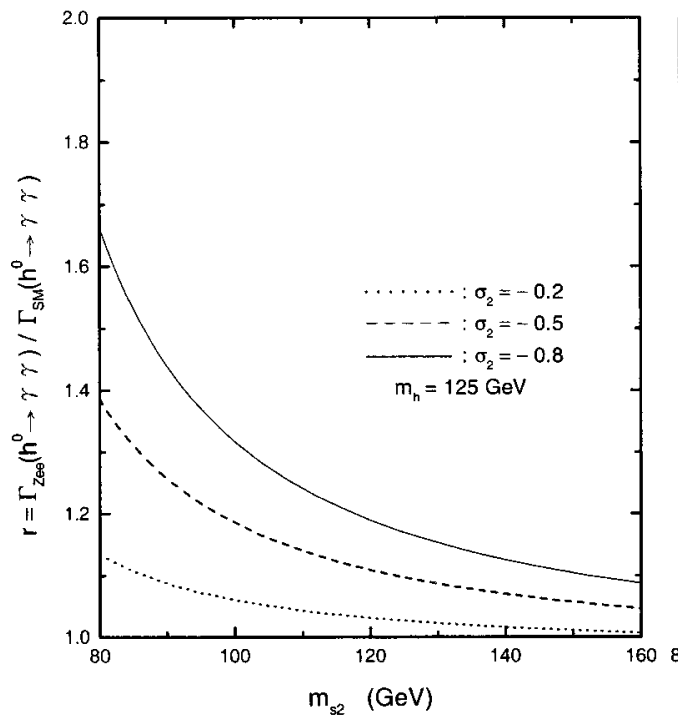

(a)

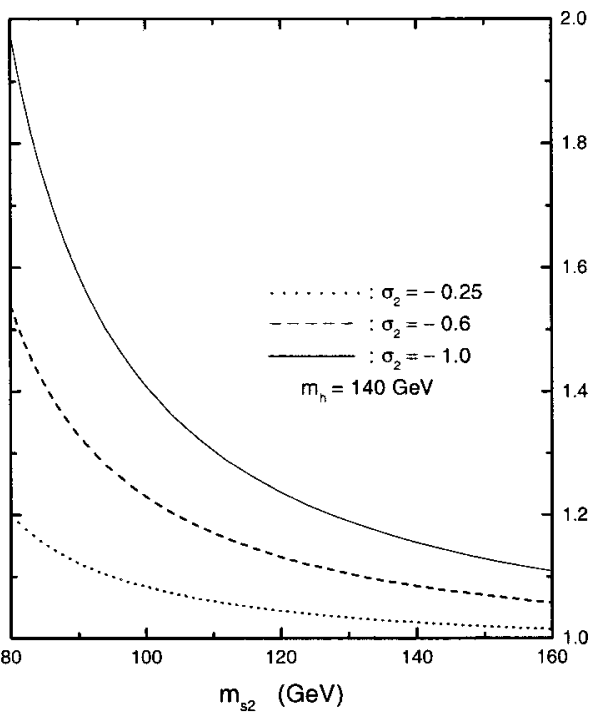

(b) similar reduction rate in $\Gamma_{\text {Zee }}(h \rightarrow \gamma \gamma)$ for a heavier $S_{2}$, the coupling constant $\sigma_{2}$ (and $\sigma_{1}$ ) has to be larger. Next, as shown in Figs. 7 and $8, \sigma_{1}$ and $\sigma_{2}$ do not have to take the same values in general, and they can be less than zero. In the case where both $\sigma_{1}$ and $\sigma_{2}$ are negative, the contributions of the $S_{2}$ loop diagram and that of the $W$ loop diagram have the same sign, so that $r$ can be larger than 1. Such an example is shown in Fig. 11(a), where the ratio $r$ for $m_{h}=125 \mathrm{GeV}$ is shown as a function of $m_{S_{2}}$ at various negative $\sigma_{2}$ values with $\sigma_{1}=0$ and $\tan \beta=16 \sqrt{2}$. We consider the case with $\sigma_{2}$ $=-0.2,-0.5$, or -0.8 , consistent with the cutoff scales $\Lambda$ $=10^{19}, 10^{7}$, or $10^{4} \mathrm{GeV}$, respectively. In the case of $\Lambda$ $=10^{19} \mathrm{GeV}\left(10^{4} \mathrm{GeV}\right)$, the deviation from the SM prediction can be about $+6 \%(+30 \%)$ for $m_{S_{2}}=100 \mathrm{GeV}$. In Fig. 11(b), a similar plot of the ratio $r$ is shown for $m_{h}$ $=140 \mathrm{GeV}$ with $\sigma_{1}=0$ and $\tan \beta=16 \sqrt{2}$. The cases with $\sigma_{2}=-0.25,-0.6$, and -1 are consistent with $\Lambda=10^{19}, 10^{7}$, and $10^{4} \mathrm{GeV}$, respectively. The correction is larger in the case with $m_{h}=140 \mathrm{GeV}$ than in the case with $m_{h}$ $=125 \mathrm{GeV}$ for a given $\Lambda$. The deviation from the SM prediction can amount to about $+8 \%(+40 \%)$ for $\Lambda$ $=10^{19} \mathrm{GeV}\left(10^{4} \mathrm{GeV}\right)$ when $m_{S_{2}}=100 \mathrm{GeV}$. Larger positive corrections are obtained for smaller $m_{S_{2}}$ values. Such a deviation from the $\mathrm{SM}$ prediction can be tested at the $\mathrm{LHC}$, the $e^{+} e^{-} \mathrm{LC}$, and the $\gamma \gamma$ option of the LC.

Before concluding this section, we remark that, if $m_{h}$ is larger than $2 m_{S_{2}}$ such that the decay mode $h \rightarrow S_{2}^{+} S_{2}^{-}$is open, the total decay width of $h$ can be greatly modified from the SM prediction for large $\sigma_{1,2}$. In terms of $R_{S_{2}}$, the partial decay width of $h \rightarrow S_{2}^{+} S_{2}^{-}$is given by

$$
\Gamma\left(h \rightarrow S_{2}^{+} S_{2}^{-}\right)=\frac{c^{2} v^{2}}{16 \pi m_{h}} \sqrt{1-4 m_{S_{2}}^{2} / m_{h}^{2}},
$$

where $c^{2}=\left(2 m_{S_{2}}^{2} R_{S_{2}} / v^{2}\right)^{2}$. In Fig. 12(a), we show the partial decay width $\Gamma\left(h \rightarrow S_{2}^{+} S_{2}^{-}\right)$for $m_{S_{2}}=80,100,150,200$
$\mathrm{GeV}$ with $\sigma_{1}=\sigma_{2}=1$ [cf. Eq. (42)] for the allowed range of $m_{h}$ from 100 to $500 \mathrm{GeV}$. In Fig. 12(b), the ratio of $\Gamma(h$ $\rightarrow S_{2}^{+} S_{2}^{-}$) to the total width of the SM Higgs boson $\left[\Gamma_{h}^{\text {total }}(\mathrm{SM})\right]$ is shown as a function of $m_{h}$ for each value of $m_{S_{2}}$. This is to illustrate the possible size of the difference between the total width of the lightest $C P$-even Higgs boson
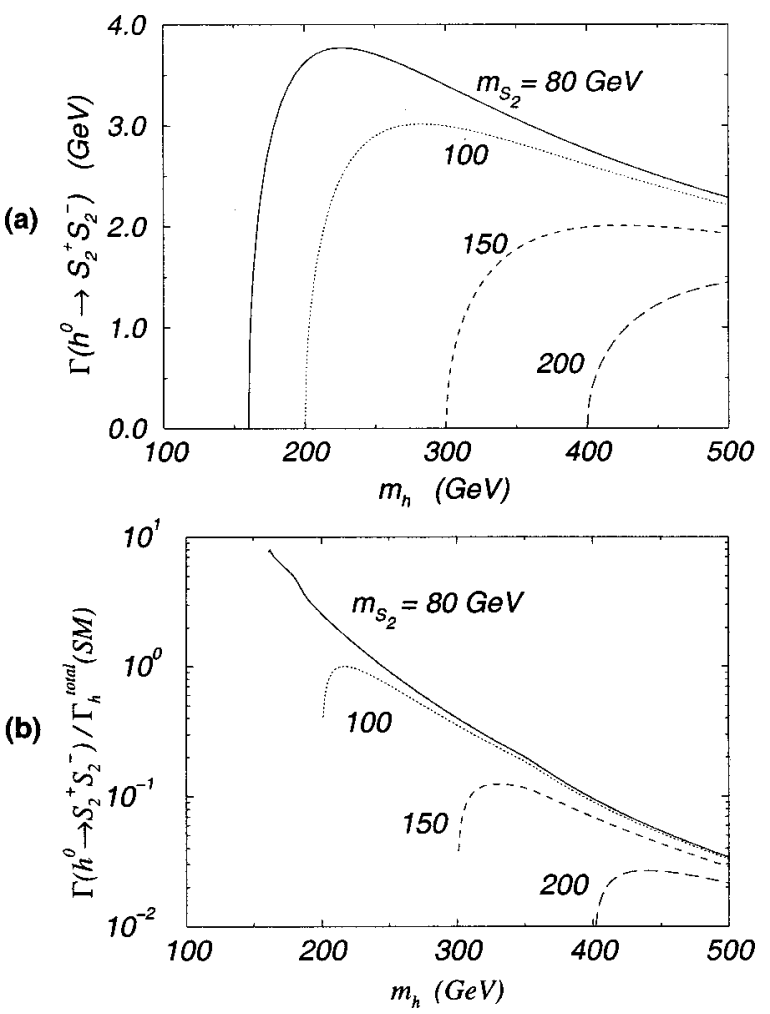

FIG. 12. (a) The partial decay width $\Gamma\left(h \rightarrow S_{2}^{+} S_{2}^{-}\right)$for $m_{S_{2}}$ $=80,100,150,200 \mathrm{GeV}$ with $\sigma_{1}=\sigma_{2}=1$ for the allowed range of $m_{h}$ from 100 to $500 \mathrm{GeV}$. (b) The ratio of $\Gamma\left(h \rightarrow S_{2}^{+} S_{2}^{-}\right)$with the total decay width of the SM Higgs boson for each value of $m_{S_{2}}$. 
$h$ in the Zee model and that of the SM Higgs boson. ${ }^{11}$ Clearly, the impact of the $S_{2}^{+} S_{2}^{-}$decay channel is especially large in the small $m_{h}$ region. We note that $\Gamma_{h}^{\text {total }}(\mathrm{SM})$ can be determined to the accuracy of 10-20\% at the LHC and the LC if $m_{h}<2 m_{Z}$, and to within a few percent if $m_{h}>2 m_{Z}$ [23]. ( $m_{Z}$ is the mass of the $Z$ boson.) Hence, measuring the total width of the lightest neutral Higgs boson can provide a further test of the Zee model for $m_{h}>2 m_{S_{2}}$. The change in the total width also modifies the decay branching ratio of $h$ $\rightarrow Z Z$, hence yielding a different rate of $h \rightarrow Z Z$ $\rightarrow \mu^{+} \mu^{-} \mu^{+} \mu^{-}$for a given $m_{h}$. (In the SM, the branching ratio of $h \rightarrow Z Z$ is about $\frac{1}{3}$ for $m_{h}>200 \mathrm{GeV}$.) Needless to say, for $m_{h}>2 m_{S_{2}}$, the production mode of $h \rightarrow S_{2}^{+} S_{2}^{-}$ $\rightarrow \ell^{+} \ell^{\prime-} \boldsymbol{E}_{T}$ is also useful to test the Zee model. Further discussion of this possibility will be given in Sec. VI.

\section{PHENOMENOLOGY OF CHARGED HIGGS BOSONS}

In the Zee model, two kinds of charged Higgs boson appear. If there is no mixing between them $(\chi=0)$, the mass eigenstates $S_{1}^{ \pm}$and $S_{2}^{ \pm}$correspond to the THDM-like charged Higgs field and the singlet Higgs field $\omega^{ \pm}$, respectively. The case with $\chi=0$ occurs in the limit of $M^{2} \gg v^{2}$, $\mu^{2}$, and $m_{0}^{2}$; i.e., in the decoupling limit. The detection of $S_{2}^{ \pm}$ can be a clear indication of the Zee model. As we will show later, its phenomenology is found to be drastically different from that of the THDM-like charged Higgs bosons $S_{1}^{ \pm}$[24]. Here, we discuss how the effects of this extra charged boson can be explored experimentally. We first consider the case with $\chi=0$, and then extend the discussion to the case with a nonzero $\chi$.

The $S_{2}^{-}$boson decays into a lepton pair $e_{i}^{-} \bar{\nu}_{e_{j}}^{c}$ with the coupling constant $f_{i j}$. The partial decay rate $\Gamma_{i j}^{S_{2}}=\Gamma\left(S_{2}^{-}\right.$ $\left.\rightarrow e_{i}^{-} \bar{\nu}_{e_{j}}^{c}\right)$ is calculated as

$$
\Gamma_{i j}^{S_{2}}=\frac{m_{S_{2}}}{4 \pi} f_{i j}^{2}\left(1-\frac{m_{e_{i}}^{2}}{m_{S_{2}}^{2}}\right)^{2},
$$

and the total decay width of $S_{2}^{-}$is given by

$$
\Gamma_{\text {total }}^{S_{2}}=\sum_{i, j=1}^{3} \Gamma_{i j}^{S_{2}} \text {. }
$$

By taking into account the hierarchy pattern of $f_{i j}$ [cf. Eqs. (23) and (24)] and by assuming $m_{S_{2}}=100 \mathrm{GeV}$ and $\left|f_{12}\right|$ $=3 \times 10^{-4}$, the total decay width and the lifetime $(\tau)$ are estimated to be ${ }^{12}$

\footnotetext{
${ }^{11}$ In doing this analysis, we have in mind a low cutoff scale $\Lambda$ $=10^{4} \mathrm{GeV}$, which allows a wide range of values for $\sigma, m_{S_{2}}$, and $m_{h}$.

${ }^{12}$ The size of the decay width depends on the value of $f_{12}$. If we take $m_{S_{1}}>500 \mathrm{GeV}$ or $\mu<100 \mathrm{GeV}, f_{12}$ can become one order of magnitude larger than $3 \times 10^{-4}$, while still being consistent with the phenomenological bounds discussed in Sec. II.
}

$$
\begin{gathered}
\Gamma_{\text {total }}^{S_{2}} \sim \Gamma_{12}^{S_{2}}+\Gamma_{21}^{S_{2}} \sim 1.6 \mathrm{keV}, \\
\tau \sim 1 / \Gamma_{\text {total }}^{S_{2}} \sim 10^{-18} \mathrm{sec} .
\end{gathered}
$$

This implies that $S_{2}$ decays after traveling a distance of $\sim 10^{-10} \mathrm{~m}$, which is significantly shorter than the typical detector scale. Therefore, $S_{2}^{ \pm}$decays promptly after its production, and can be detected in collider experiments.

The main production channel at the LEP-II experiment may be the pair production process $e^{+} e^{-} \rightarrow S_{2}^{+} S_{2}^{-}$, similar to the production of the THDM-like charged Higgs boson $S_{1}^{+}$. The matrix-element squares for $S_{i}^{+} S_{i}^{-}$production $(i$ $=1,2$ ) are given by

$$
\begin{aligned}
\mid \mathcal{M}( & \left.e_{L(R)}^{-1} e_{R(L)}^{+} \rightarrow S_{i}^{+} S_{i}^{-}\right)\left.\right|^{2} \\
= & \left\{\frac{Q_{e} e^{2}}{s}-\frac{1}{c_{W}^{2}}\left(I_{S_{i}}^{3}-s_{W}^{2} Q_{S_{i}}\right) \frac{\left(I_{e}^{3}-s_{W}^{2} Q_{e}\right) g^{2}}{s-m_{Z}^{2}}\right\}^{2} \\
& \times s^{2} \beta_{S_{i}}^{2} \sin ^{2} \Theta
\end{aligned}
$$

where $Q_{e}=-1$ and $I_{e}^{3}=-\frac{1}{2}(0)$ for the incoming electron $e_{L}^{-}\left(e_{R}^{-}\right) ; \quad Q_{S_{i}}=-1$ and $I_{S_{i}}^{3}=-\frac{1}{2}(0)$ for $i=1$ (2); $\beta_{S_{i}}$ $=\sqrt{1-4 m_{S_{i}}^{2} / s}, s_{W}=\sin \theta_{W}, c_{W}=\cos \theta_{W}$, and $\Theta$ is the scattering angle of $S_{i}^{-}$in the $e^{+} e^{-}$center-of-mass (c.m.) frame whose energy is $\sqrt{s}$. For the other electron-positron helicity configuration $\left(e_{L}^{-} e_{L}^{+}\right.$and $\left.e_{R}^{-} e_{R}^{+}\right)$, the cross sections are zero. Thus the total cross section for the $S_{2}^{+} S_{2}^{-}$pair production is given by

$$
\begin{aligned}
\sigma\left(e^{+} e^{-} \rightarrow S_{2}^{+} S_{2}^{-}\right)= & \frac{1}{96 \pi} e^{4} \beta_{S_{2}}^{3} s\left[\left(\frac{1}{s}+\frac{s_{W}^{2}}{c_{W}^{2}} \frac{1}{s-m_{Z}^{2}}\right)^{2}\right. \\
& \left.+\left\{\frac{1}{s}-\left(\frac{1}{2}-s_{W}^{2}\right) \frac{1}{c_{W}^{2}} \frac{1}{s-m_{Z}^{2}}\right\}^{2}\right]
\end{aligned}
$$

Hence, the production rates of $S_{1}^{-}$and $S_{2}^{-}$are different. We note that the ratio of cross sections for $S_{1}^{+} S_{1}^{-}$and $S_{2}^{+} S_{2}^{-}$ production, $\sigma\left(e^{+} e^{-} \rightarrow S_{2}^{+} S_{2}^{-}\right) / \sigma\left(e^{+} e^{-} \rightarrow S_{1}^{+} S_{1}^{-}\right)$, is 0.8 at $\sqrt{s}=210 \mathrm{GeV}$, assuming that the masses of $S_{1}^{ \pm}$and $S_{2}^{ \pm}$are the same. This ratio is independent of the masses of $S_{1}$ and $S_{2}$ for a fixed c.m. energy. (Only the difference between $S_{1}^{+} S_{1}^{-} Z$ and $S_{2}^{+} S_{2}^{-} Z$ coupling constants determines this ratio.)

The lower mass bound of the THDM-like charged boson $S_{1}^{ \pm}$can be obtained by studying its $\tau \nu$ and $c s$ decay modes, completely in the same way as the charged Higgs boson search in the minimal supersymmetric standard model (MSSM) [22]. Similar experimental constraints may be obtained for the extra charged bosons $S_{2}^{ \pm}$. The situation, however, turns out to be fairly different from the $S_{1}^{ \pm}$case. First of all, decays of $S_{2}^{ \pm}$are all leptonic. Secondly, the branching ratios of various $S_{2}^{ \pm}$decay modes are estimated as

$$
B\left(S_{2}^{-} \rightarrow e^{-} \boldsymbol{E}_{T}\right) \sim 0.5
$$




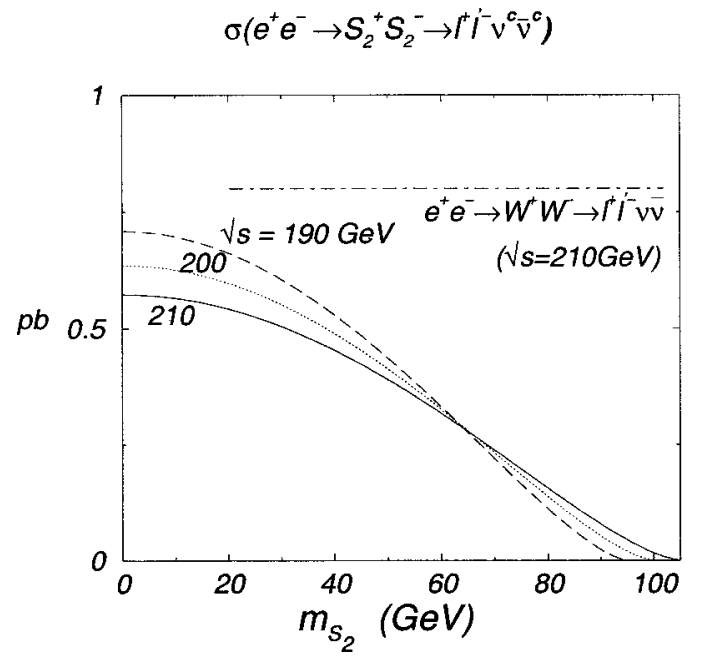

FIG. 13. The cross section of the leptonic decay process $e^{+} e^{-}$ $\rightarrow S_{2}^{+} S_{2}^{-} \rightarrow \ell^{+} \ell^{\prime}-\boldsymbol{E}_{T}$ (where $\ell$ and $\ell^{\prime}=e$ or $\mu$ ) at $\sqrt{s}=190,200$, and $210 \mathrm{GeV}$. The process $e^{+} e^{-} \rightarrow W^{+} W^{-} \rightarrow \ell^{+} \ell^{\prime-} \boldsymbol{E}_{T}$ at $\sqrt{s}$ $=210 \mathrm{GeV}$ is shown for comparison.

$$
\begin{aligned}
& B\left(S_{2}^{-} \rightarrow \mu^{-} \boldsymbol{E}_{T}\right) \sim 0.5, \\
& B\left(S_{2}^{-} \rightarrow \tau^{-} \boldsymbol{E}_{T}\right) \sim \mathcal{O}\left(\frac{m_{\mu}^{4}}{m_{\tau}^{4}}\right) \sim 10^{-5},
\end{aligned}
$$

where we have used the relations given in Eqs. (23) and (24). Clearly, the branching ratio into the $\tau^{-} \boldsymbol{E}_{T}$ mode is very small, so that it is not useful for detecting $S_{2}^{ \pm}$at all. This is different from the case of detecting the ordinary THDM-like charged Higgs boson, which preferentially decays into heavy fermion pairs (e.g., $\tau \nu$ and $c s$ ). Instead of studying the $\tau^{ \pm} \nu^{c}$ mode, the $e^{ \pm} \nu^{c}$ and $\mu^{ \pm} \nu^{c}$ modes can provide a strong constraint on the mass of $S_{2}^{ \pm}$. In fact, the branching ratio of $S_{2}^{-} \rightarrow e^{-} \boldsymbol{E}_{T}$ or $\mu^{-} \boldsymbol{E}_{T}$ is almost $100 \%$, so that we have $\sigma\left(e^{+} e^{-} \rightarrow S_{2}^{+} S_{2}^{-} \rightarrow \ell^{+} \ell^{\prime-} \boldsymbol{E}_{T}\right) \sim \sigma\left(e^{+} e^{-} \rightarrow S_{2}^{+} S_{2}^{-}\right)$, where $\ell^{-}$and $\ell^{\prime-}$ represent $e^{-}$or $\mu^{-}$(not $\tau^{-}$). Let us compare this with the cross section $\sigma\left(e^{+} e^{-} \rightarrow W^{+} W^{-} \rightarrow \ell^{+} \ell^{\prime-} \boldsymbol{E}_{T}\right)$ $=\sigma\left(e^{+} e^{-} \rightarrow W^{+} W^{-}\right) B\left(W^{-} \rightarrow \ell^{-} \boldsymbol{E}_{T}\right)^{2}, \quad$ where $B\left(W^{-}\right.$ $\left.\rightarrow \ell^{-} \boldsymbol{E}_{T}\right)=B\left(W^{-} \rightarrow e^{-} \boldsymbol{E}_{T}\right)+B\left(W^{-} \rightarrow \mu^{-} \boldsymbol{E}_{T}\right) \sim 21 \%$. As seen in Fig. 13, the cross section $\sigma\left(e^{+} e^{-} \rightarrow S_{2}^{+} S_{2}^{-}\right.$ $\left.\rightarrow \ell^{+} \ell^{\prime-} \mathbb{E}_{T}\right)$ is comparable with $\sigma\left(e^{+} e^{-} \rightarrow W^{+} W^{-}\right.$ $\left.\rightarrow \ell^{+} \ell^{\prime-} \boldsymbol{E}_{T}\right)$. Therefore, by examining the LEP-II data for $\ell^{+} \ell^{\prime-} \boldsymbol{E}_{T}$ (where $\ell^{+} \ell^{\prime-}=e^{+} e^{-}, e^{ \pm} \mu^{\mp}$, or $\mu^{+} \mu^{-}$, in contrast to $\tau^{+} \tau^{-}$for the $S_{1}^{ \pm}$case), the experimental lower bound on the mass of $S_{2}^{ \pm}$can be determined. Such a bound can be induced from the smuon search results at the LEP experiments $[25,26]$ in the case that neutralinos are assumed to be massless. From the $\mu^{+} \mu^{-} \boldsymbol{E}_{T}$ data accumulated up to $\sqrt{s}=202 \mathrm{GeV}$ [26], we find that the lower mass bound of $S_{2}^{ \pm}$ is likely to be $80-85 \mathrm{GeV}$ for the $\chi=0$ cases. [We note that the right-handed smuon $\left(\widetilde{\mu}_{R}^{ \pm}\right)$in the MSSM carries the same $\mathrm{SU}(2) \times U(1)$ quantum number as the weak-singlet charged Higgs boson $\left(S_{2}^{ \pm}\right.$for $\left.\chi^{ \pm} \sim 0\right)$.]

We next comment on $S_{2}^{ \pm}$production processes at hadron colliders and future LC's. At hadron colliders, the dominant

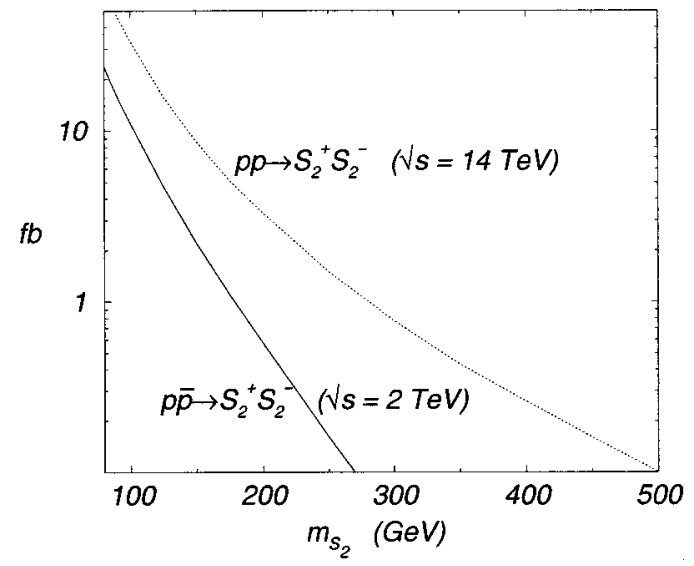

FIG. 14. The total cross sections of $p \bar{p} \rightarrow S_{2}^{+} S_{2}^{-}$at $\sqrt{s}=2 \mathrm{TeV}$ (solid curve) and $p p \rightarrow S_{2}^{+} S_{2}^{-}$at $\sqrt{s}=14 \mathrm{TeV}$ (dotted curve) as functions of $m_{S_{2}}$.

production mode is pair production through the Drell-Yantype process. The cross sections for $p \bar{p} \rightarrow S_{2}^{+} S_{2}^{-}$at the Tevatron run-II energy $(\sqrt{s}=2 \mathrm{TeV})$ and $p p \rightarrow S_{2}^{+} S_{2}^{-}$at the LHC energy $(\sqrt{s}=14 \mathrm{TeV})$ are shown as functions of $m_{S_{2}}$ in Fig. 14 for $\chi=0$. At future LC's, the $S_{2}^{ \pm}$boson may be discovered through the above-discussed pair-production process from electron-positron annihilation if $\sqrt{s} / 2>m_{S_{2}}$. In Fig. 15, we show the total cross section of $e^{+} e^{-} \rightarrow S_{2}^{+} S_{2}^{-}$for $\chi=0$ as a function of $m_{S_{2}}$ for $\sqrt{s}=300,500$, and $1000 \mathrm{GeV}$.

Finally, we wish to discuss the case with a nonzero $\chi$, in which $S_{2}^{-}$is a mixture of the singlet charged Higgs boson state $\left(\omega^{-}\right)$and the doublet charged Higgs boson state $\left(H^{-}\right)$. Let us see how the above discussion is changed in this case. The doublet charged Higgs bosons with mass of $100 \mathrm{GeV}$ mainly decay into the $\tau^{-} \nu$ and $\bar{c} s$ channels. Thus, the branching ratio of the decay process $S_{2}^{-} \rightarrow \ell^{-} \boldsymbol{E}_{T}$, where $\ell^{-}$ represents $e^{-}$and $\mu^{-}$, is expressed in the nonzero $\chi$ case as

$$
B\left(S_{2}^{-} \rightarrow \ell^{-} \boldsymbol{E}_{T}\right)=\frac{\left.\cos ^{2} \chi \Gamma_{\text {total }}^{S_{2}}\right|_{\chi=0}}{\left.\sin ^{2} \chi \Gamma_{\text {total }}^{S_{1}}\right|_{\chi=0}+\left.\cos ^{2} \chi \Gamma_{\text {total }}^{S_{2}}\right|_{\chi=0}},
$$

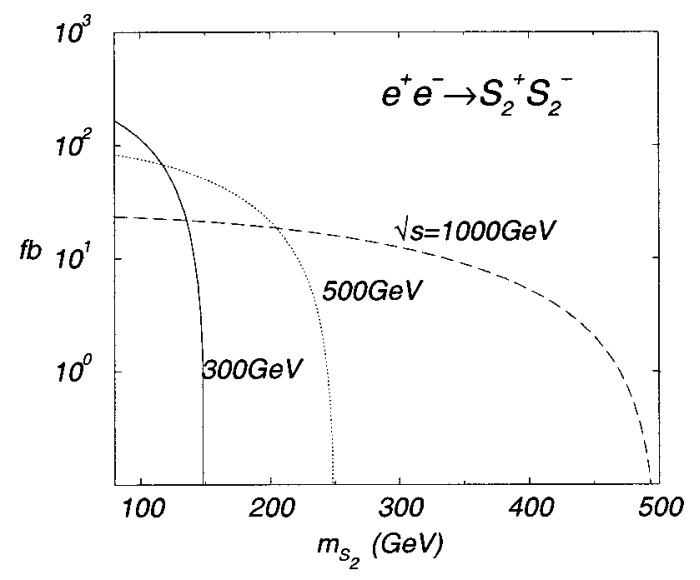

FIG. 15. The total cross section of $e^{+} e^{-} \rightarrow S_{2}^{+} S_{2}^{-}$as a function of $m_{S_{2}}$ at $\sqrt{s}=300,500$, and $1000 \mathrm{GeV}$. 


$$
B\left(S_{2}^{-} \rightarrow l^{-} v\right)
$$

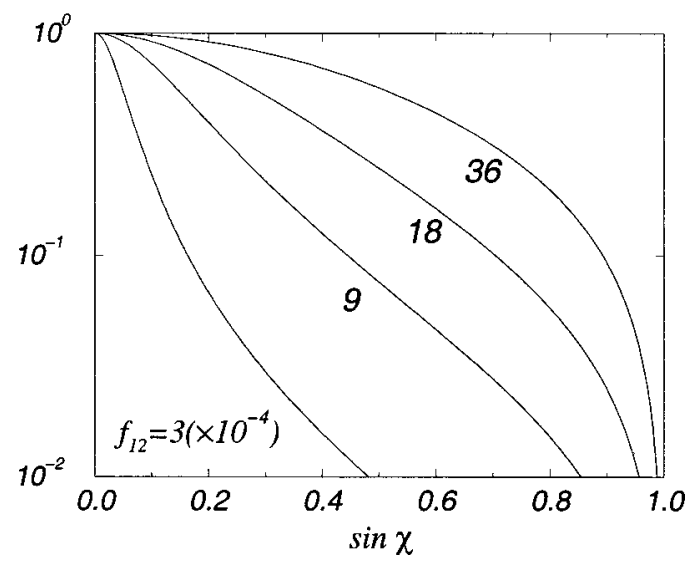

FIG. 16. The decay branching ratio of $S_{2}^{-} \rightarrow \ell^{-} \mathbb{E}_{T}$ (where $\ell^{-}$ $=e^{-}$or $\left.\mu^{-}\right)$as a function of the mixing angle $\chi$ for $m_{S_{2}}$ $=100 \mathrm{GeV}, \tan \beta=1$, and various values of the coupling constant $f_{12}$.

where $\left.\Gamma_{\text {total }}^{S_{i}}\right|_{\chi=0}(i=1,2)$ is the total width of $S_{i}^{-}$at $\chi=0$ with the same mass as the decaying $S_{2}^{-}$on the left-hand side of the above equation. The formula of $\left.\Gamma_{\text {total }}^{S_{2}}\right|_{\chi=0}$ is given in Eq. (45), while $\left.\Gamma_{\text {total }}^{S_{1}}\right|_{\chi=0}$, which is the same as the total decay width of the charged Higgs boson in the THDM, is given by

$$
\left.\Gamma_{\text {total }}^{S_{1}}\right|_{\chi=0}=\sum_{\bar{f} f^{\prime}} \Gamma\left(S_{1}^{-} \rightarrow \bar{f} f^{\prime}\right),
$$

where $\bar{f} f^{\prime}$ are fermion pairs which are kinematically allowed. In the type-II Yukawa couplings, we have

$$
\begin{aligned}
\Gamma\left(S_{1}^{-} \rightarrow \tau^{-} \nu\right) & =\frac{m_{S_{1}}}{8 \pi v^{2}}\left(m_{\tau}^{2} \tan ^{2} \beta\right)\left(1-\frac{m_{\tau}^{2}}{m_{S_{1}}^{2}}\right)^{2}, \\
\Gamma\left(S_{1}^{-} \rightarrow \bar{c} s\right) & \simeq \frac{3 m_{S_{1}}}{8 \pi v^{2}}\left(m_{s}^{2} \tan ^{2} \beta+m_{c}^{2} \cot ^{2} \beta\right)\left(1-\frac{m_{c}^{2}}{m_{S_{1}}^{2}}\right)^{2}, \\
\Gamma\left(S_{1}^{-} \rightarrow \bar{t} b\right) & \simeq \frac{3 m_{S_{1}}}{8 \pi v^{2}}\left(m_{b}^{2} \tan ^{2} \beta+m_{t}^{2} \cot ^{2} \beta\right)\left(1-\frac{m_{t}^{2}}{m_{S_{1}}^{2}}\right)^{2} .
\end{aligned}
$$

In the THDM, the total decay width of the charged Higgs boson $\left(H^{-}\right)$for $m_{H^{-}}=100 \mathrm{GeV}$ is about $470 \mathrm{keV}$. Hence, if the mixing angle $\chi$ is not too small, the decay pattern of $S_{2}^{-}$ is dominated by that of the THDM charged Higgs boson $H^{-}$. In Fig. 16, we plot the branching ratio $B\left(S_{2}^{-} \rightarrow l^{-} \boldsymbol{E}_{T}\right)$ as a function of $\sin \chi$ at $m_{S_{2}}=100 \mathrm{GeV}$ for several values of $f_{12}$. We show only the case with $\tan \beta=1$, where the result is independent of the type of Yukawa interaction. The coupling constant $f_{12}$ is taken to be $3,9,18$, and $36\left(\times 10^{-4}\right)$, which satisfy the phenomenological constraints given in Sec. II. As expected, the branching ratio decreases as $\chi$ increases. When $f_{12}=36 \times 10^{-4}, B\left(S_{2}^{-} \rightarrow l^{-} \boldsymbol{E}_{T}\right)$ is smaller than $10 \%$ for $\sin \chi>0.89$. For the smaller $f_{12}$ values, the branching ratio is reduced more quickly. The branching ratio is not sensitive to $m_{S_{2}}$ unless the mass exceeds the threshold of decay into a $\bar{t} b$ or $h^{0} W^{-}$pair. Above the threshold of $\bar{t} b$ pair production, the decay rate of $S_{2}^{-} \rightarrow \bar{t} b$ is large due to the large mass of the top quarks, so that $B\left(S_{2}^{-} \rightarrow l^{-} \boldsymbol{E}_{T}\right)$ is substantial only for very small values of $\chi$. Finally, while the decay branching ratio can change drastically depending on the mixing angle $\chi$, the production cross section for $e^{+} e^{-} \rightarrow S_{2}^{+} S_{2}^{-}$remains unchanged. In conclusion, the process $e^{+} e^{-} \rightarrow S_{2}^{+} S_{2}^{-}$ $\rightarrow l^{+} l^{\prime-} \boldsymbol{E}_{T}$ can also be useful for testing the Zee model in the nonzero $\chi$ case, provided $\sin \chi$ is not too large.

\section{DISCUSSION AND CONCLUSION}

In this paper, the Higgs sector of the Zee model has been investigated, in which neutrino masses are generated radiatively. This model contains an extra weak-doublet Higgs field and singlet charged Higgs field.

We have studied indirect effects of these extra Higgs bosons on the theoretical mass bounds of the lightest $C P$ even Higgs boson, which are obtained from the requirement that the running coupling constants neither blow up to a very large value nor decrease to a negative value, up to a high energy cutoff scale $\Lambda$. For $\Lambda=10^{19} \mathrm{GeV}$, the upper bound of $m_{h}$ is found to be about $175 \mathrm{GeV}$, which is almost the same value as the SM prediction. In the decoupling regime $(M$ $\gg m_{Z}$ ), the lower bound is found to be about $100 \mathrm{GeV}$ for $\Lambda=10^{19} \mathrm{GeV}$, which is much smaller than the lower bound in the SM, and is almost the same as that in the THDM. For smaller $\Lambda$ values, the bounds are more relaxed, similar to that of the SM. We have also investigated the allowed range of coupling constants relevant to the weak-singlet Higgs field.

The most striking feature of the Zee model Higgs sector is the existence of the weak-singlet charged Higgs boson. We have examined the possible impact of the singlet charged Higgs boson on the neutral Higgs boson search through radiative corrections. We found that its one-loop contributions to the $h \rightarrow \gamma \gamma$ width can be sizable. In the allowed range of coupling constants the deviation from the SM prediction for this decay width can be about $-20 \%$ or near $+10 \%$ for $m_{S_{2}}=100 \mathrm{GeV}$ and $\Lambda=10^{19} \mathrm{GeV}$, depending on the sign of the coupling constants $\sigma_{i}$. The magnitude of the deviation is larger for lower $\Lambda$ values or for smaller $m_{S_{2}}$ values. For example, a positive deviation over $30-40 \%$ is possible for $m_{h}=125-140 \mathrm{GeV}, m_{S_{2}}=100 \mathrm{GeV}$, and $\Lambda=10^{4} \mathrm{GeV}$.

In the decoupling limit (i.e., when $M^{2} \gg v^{2}$, where $\alpha$ $\rightarrow \beta-\pi / 2$ and $\chi \rightarrow 0$ ), we expect the production cross sections for $g g \rightarrow h, e^{+} e^{-} \rightarrow \nu \bar{\nu} h$, and $e^{+} e^{-} \rightarrow Z^{0} h$ in the Zee model to be the same as those in the SM. However, a sizable change in the decay branching ratio of $h \rightarrow \gamma \gamma$ can alter the production rate of $p p \rightarrow h X \rightarrow \gamma \gamma X$ at the LHC, where this production rate can be determined with a relative error of $10-15 \%$ [15]. Also, such a deviation in the branching ratio of $h \rightarrow \gamma \gamma$ directly affects the cross section of $e^{+} e^{-} \rightarrow \nu \bar{\nu} h$ 
(and $\left.Z^{0} h\right) \rightarrow \nu \bar{\nu} \gamma \gamma$, which can be measured with an accuracy of $16-22 \%$ at future $e^{+} e^{-}$LC's (with $\sqrt{s}=500 \mathrm{GeV}$ and the integrated luminosity of $1 \mathrm{ab}^{-1}$ ) [16]. Therefore, the Zee model with low cutoff scales can be tested through the $h \rightarrow \gamma \gamma$ process at the LHC and $e^{+} e^{-}$LC's. At future photon colliders, the enhancement (or reduction) of the $h \rightarrow \gamma \gamma$ partial decay rate will manifest itself in a different production rate of $h$ from the SM prediction. A few percent of the deviation in $\Gamma(h \rightarrow \gamma \gamma) B(h \rightarrow b \bar{b})$ can be detected at a photon collider [17], so that the effects of the singlet charged Higgs boson can be tested even if the cutoff scale $\Lambda$ is at the Planck scale.

The collider phenomenology of the singlet charged Higgs boson has turned out to be completely different from that of the THDM-like charged Higgs boson. The singlet charged Higgs boson mainly decays into $l^{ \pm} \boldsymbol{E}_{T}$ (with $l^{ \pm}=e^{ \pm}$or $\mu^{ \pm}$), while the decay mode $\tau^{ \pm} \boldsymbol{E}_{T}$ is almost negligible due to the relation $\left|f_{12}\right| \gg\left|f_{13}\right| \gg\left|f_{23}\right|$. This hierarchy among the coupling constants $f_{i j}$ results from demanding bimaximal mixings in the neutrino mass matrix generated in the Zee model to be consistent with the neutrino oscillation data. On the other hand, the THDM-like charged Higgs boson decays mainly into either the $\tau \nu$ mode or the $c s$ mode, through the usual Yukawa interactions. Hence, to probe this singlet charged Higgs boson using the LEP-II data, experimentalists should examine their data sample with $e^{+} e^{-} \boldsymbol{E}_{T}, e^{+} \mu^{-} \boldsymbol{E}_{T}$, $\mu^{+} e^{-} \boldsymbol{E}_{T}$, or $\mu^{+} \mu^{-} \boldsymbol{E}_{T}$, while the experimental lower mass bound of the THDM-like charged Higgs boson is obtained from examining the $\tau \tau \boldsymbol{E}_{T}, \tau \boldsymbol{E}_{T j} j$, and jjjj events. Using the published LEP-II constraints on the MSSM smuon production (assuming the lightest neutralinos to be massless), we estimate the current lower mass bound of this singlet charged Higgs boson to be about $80-85 \mathrm{GeV}$. The Tevatron run II, LHC, and future LC's can further test this model.

Finally, we comment on a case in which the singlet charged Higgs boson $\left(S_{2}^{ \pm}\right.$for $\left.\chi=0\right)$ is the lightest of all the Higgs bosons. For $m_{h} / 2>m_{S_{2}}>m_{Z}$, the Higgs sector of the Zee model can be further tested by measuring the production rate of $p p($ or $p \bar{p}) \rightarrow h X \rightarrow S_{2}^{+} S_{2}^{-} X \rightarrow l^{+} l^{\prime-} \boldsymbol{E}_{T} X$. The branching ratio for $h \rightarrow S_{2}^{+} S_{2}^{-} \rightarrow \ell^{+} \ell^{\prime-} \boldsymbol{E}_{T}$ can be large. For instance, for $m_{h}=210 \mathrm{GeV}$ and $m_{S_{2}}=100 \mathrm{GeV}$, this branching ratio is about $12 \%$ for each $\ell^{+} \ell^{\prime-}=e^{+} e^{-}, e^{+} \mu^{-}, \mu^{+} e^{-}$, or $\mu^{+} \mu^{-}$. The branching ratio decreases for larger masses of $h$. Moreover, the total decay width of $h$ can be greatly modified when the decay channel $h \rightarrow S_{2}^{+} S_{2}^{-}$is open. In this case, the decay branching ratios of $h \rightarrow W^{+} W^{-}, Z Z$ are also different from the SM predictions.

In conclusion, the features distinguishing the Zee model from the SM and the THDM can be tested by the data from LEP-II, the Tevatron run II, and future experiments at the LHC and LC's.

\section{ACKNOWLEDGMENTS}

We are grateful for the warm hospitality of the Center for Theoretical Sciences in Taiwan where part of this work was completed. C.-P.Y. would like to thank H.-J. He, J. Ng, and W. Repko for stimulating discussions. S.K. was supported, in part, by the Alexander von Humboldt Foundation. G.-L.L. and J.-J.T. were supported, in part, by the National Science Council of R.O.C. under the Grant No. NSC-89-2112-M009-035; Y.O. was supported by a Grant-in-Aid of the Ministry of Education, Science, Sports and Culture, Government of Japan (No. 09640381), Priority area "Supersymmetry and Unified Theory of Elementary Particles" (No. 707), and "Physics of CP Violation" (No. 09246105); C.-P.Y. was supported by the U.S. National Science Foundation under Grant PHY-9802564.

\section{APPENDIX: ONE-LOOP RGE'S FOR DIMENSIONLESS COUPLING CONSTANTS}

Here, we summarize the RGE's relevant to our study. For the gauge coupling constants, we have

$$
\begin{aligned}
& \mu \frac{d}{d \mu} g_{1}=\frac{1}{16 \pi^{2}} \frac{22}{3} g_{1}^{3}, \\
& \mu \frac{d}{d \mu} g_{2}=\frac{1}{16 \pi^{2}}(-3) g_{2}^{3}, \\
& \mu \frac{d}{d \mu} g^{3}=\frac{1}{16 \pi^{2}}(-7) g_{3}^{3} .
\end{aligned}
$$

The RGE's for the Higgs self-coupling constants of the doublets are calculated at the one-loop level as

$$
\begin{aligned}
\mu \frac{d}{d \mu} \lambda_{1}= & \frac{1}{16 \pi^{2}}\left\{12 \lambda_{1}^{2}+4 \lambda_{3}^{2}+4 \lambda_{3} \lambda_{4}+2 \lambda_{4}^{2}+2 \lambda_{5}^{2}+2 \sigma_{1}^{2}\right. \\
& \left.-\left(3 g_{1}^{2}+9 g_{2}^{2}\right) \lambda_{1}+\left(\frac{3}{4} g_{1}^{4}+\frac{3}{2} g_{1}^{2} g_{2}^{2}+\frac{9}{4} g_{2}^{4}\right)\right\},
\end{aligned}
$$

$$
\begin{aligned}
\mu \frac{d}{d \mu} \lambda_{2}= & \frac{1}{16 \pi^{2}}\left\{12 \lambda_{2}^{2}+4 \lambda_{3}^{2}+4 \lambda_{3} \lambda_{4}+2 \lambda_{4}^{2}+2 \lambda_{5}^{2}+2 \sigma_{2}^{2}\right. \\
& +12 y_{t}^{2} \lambda_{2}-12 y_{t}^{4}-\left(3 g_{1}^{2}+9 g_{2}^{2}\right) \lambda_{2} \\
& \left.+\left(\frac{3}{4} g_{1}^{4}+\frac{3}{2} g_{1}^{2} g_{2}^{2}+\frac{9}{4} g_{2}^{4}\right)\right\}
\end{aligned}
$$

$$
\begin{aligned}
\mu \frac{d}{d \mu} \lambda_{3}= & \frac{1}{16 \pi^{2}}\left\{2\left(\lambda_{1}+\lambda_{2}\right)\left(3 \lambda_{3}+\lambda_{4}\right)+4 \lambda_{3}^{2}+2 \lambda_{4}^{2}+2 \lambda_{5}^{2}\right. \\
& +2 \sigma_{1} \sigma_{2}+6 y_{t}^{2} \lambda_{3}-\left(3 g_{1}^{2}+9 g_{2}^{2}\right) \lambda_{3} \\
& \left.+\left(\frac{3}{4} g_{1}^{4}-\frac{3}{2} g_{1}^{2} g_{2}^{2}+\frac{9}{4} g_{2}^{4}\right)\right\}
\end{aligned}
$$

$$
\begin{aligned}
\mu \frac{d}{\mu} \lambda_{4}= & \frac{1}{16 \pi^{2}}\left\{2\left(\lambda_{1}+\lambda_{2}\right) \lambda_{4}+4\left(2 \lambda_{3}+\lambda_{4}\right) \lambda_{4}+8 \lambda_{5}^{2}\right. \\
& \left.+6 y_{t}^{2} \lambda_{4}-\left(3 g_{1}^{2}+9 g_{2}^{2}\right) \lambda_{4}+3 g_{1}^{2} g_{2}^{2}\right\},
\end{aligned}
$$




$$
\begin{aligned}
\mu \frac{d}{d \mu} \lambda_{5}= & \frac{1}{16 \pi^{2}}\left\{2 \lambda_{1}+2 \lambda_{2}+8 \lambda_{3}+12 \lambda_{4}+6 y_{t}^{2}\right. \\
& \left.-\left(3 g_{1}^{2}+9 g_{2}^{2}\right)\right\} \lambda_{5},
\end{aligned}
$$

and those with respect to the additional singlet charged Higgs bosons are given by

$$
\begin{aligned}
\mu \frac{d}{d \mu} \sigma_{1}= & \frac{1}{16 \pi^{2}}\left\{4 \sigma_{1}^{2}+2 \sigma_{1} \sigma_{3}+6 \lambda_{1} \sigma_{1}+\left(4 \lambda_{3}+2 \lambda_{4}\right) \sigma_{2}\right. \\
& \left.+8 f_{i j} f_{i j} \sigma_{1}-\left(\frac{15}{2} g_{1}^{2}+\frac{9}{2} g_{2}^{2}\right) \sigma_{1}+3 g_{1}^{4}\right\}, \quad \text { (A9) } \\
\mu \frac{d}{d \mu} \sigma_{2}= & \frac{1}{16 \pi^{2}}\left\{4 \sigma_{2}^{2}+2 \sigma_{2} \sigma_{3}+6 \lambda_{2} \sigma_{2}+\left(4 \lambda_{3}+2 \lambda_{4}\right) \sigma_{1}\right. \\
& +6 y_{t}^{2} \sigma_{2}+8 f_{i j} f_{i j} \sigma_{2}-\left(\frac{15}{2} g_{1}^{2}+\frac{9}{2} g_{2}^{2}\right) \\
& \left.\times \sigma_{2}+3 g_{1}^{4}\right\}, \\
\mu \frac{d}{d \mu} \sigma_{3}= & \frac{1}{16 \pi^{2}}\left\{8 \sigma_{1}^{2}+8 \sigma_{2}^{2}+5 \sigma_{3}^{2}+16 f_{i j} f_{i j} \sigma_{3}-128 \operatorname{tr} f^{4}\right. \\
& \left.-12 g_{1}^{2} \sigma_{3}+24 g_{1}^{4}\right\} .
\end{aligned}
$$

Finally, the RGE's for the Yukawa-type coupling constants are obtained at one-loop level as

$$
\mu \frac{d}{d \mu} y_{t}=\frac{1}{16 \pi^{2}}\left\{-\left(\frac{17}{12} g_{1}^{2}+\frac{9}{4} g_{2}^{2}+8 g_{3}^{2}\right) y_{t}+\frac{9}{2} y_{t}^{3}\right\}
$$

$$
\begin{aligned}
\mu \frac{d}{d \mu} f_{i j}= & \frac{1}{16 \pi^{2}}\left\{-\left(\frac{3}{2} g_{1}^{2}+\frac{9}{2} g_{2}^{2}\right) f_{i j}\right. \\
& \left.+4 f_{k l} f_{k l} f_{i j}-4 f_{i k} f_{k l} f_{i j}\right\},
\end{aligned}
$$

where

$$
\begin{aligned}
\operatorname{tr} f^{4} & \equiv \sum_{i, j, k, l=1-3} f_{i j} f_{j k} f_{k l} f_{l i}, \\
f_{i j} f_{i j} & \equiv \sum_{i, j=1-3} f_{i j} f_{i j} .
\end{aligned}
$$

[1] Super-Kamiokande Collaboration, Y. Fukuda et al., Phys. Rev. Lett. 81, 1562 (1998).

[2] A. Zee, Phys. Lett. 93B, 339 (1980); 161B, 141 (1985).

[3] S. T. Petcov, Phys. Lett. 115B, 401 (1982); J. Liu, Phys. Lett. B 216, 367 (1989); B. K. Pal, Phys. Rev. D 44, 2261 (1991); W. Grimus and G. Nardulli, Phys. Lett. B 271, 161 (1991); A. Y. Smirnov and Z.-J. Tao, Nucl. Phys. B426, 415 (1994); P. H. Frampton and S. L. Glashow, Phys. Lett. B 461, 95 (1999); G. C. McLaughlin and J. N. Ng, ibid. 464, 232 (1999); A. S. Jaurabh and S. D. Rindani, ibid. 464, 239 (1999); K. Cheung and O. C. W. Kong, Phys. Rev. D 61, 113012 (2000); hep-ph/0003276; Y. Koide and A. Ghosal, Phys. Rev. D 63, 037301 (2001).

[4] C. Jarlskog, M. Matsuda, S. Skadhauge, and M. Tanimoto, Phys. Lett. B 449, 240 (1999).

[5] M. Matsuda, C. Jarlskog, S. Skadhauge, and M. Tanimoto, hep-ph/0005147.

[6] A. Yu. Smirnov and M. Tanimoto, Phys. Rev. D 55, 1665 (1997).

[7] G. C. McLaughlin and J. N. Ng, Phys. Lett. B 455, 224 (1999).

[8] ALEPH Collaboration, R. Barote et al., Phys. Lett. B 487, 253 (2000).

[9] "Report of the Higgs Working Group" from the Physics at Run II Supersymmetry/Higgs Workshop, hep-ph/0010338.

[10] CMS Technical Proposal No. CERN/LHCC/94-38, 1994; ATLAS Technical Proposal No. CERN/LHCC/94-93 1994; ATLAS Technical Design Report No. CERN/LHCC//99-15, 1999.

[11] N. Cabibbo, L. Maiani, G. Parisi, and R. Petronzio, Nucl.
Phys. B158, 295 (1976); M. Lindner, Z. Phys. C 31, 295 (1986); G. Altarelli and I. Ishidori, Phys. Lett. B 337, 141 (1994); J. A. Casas, J. R. Espinosa, and M. Quirós, ibid. 342, 171 (1995); 374, 382 (1996); J. R. Espinosa and M. Quirós, ibid. 353, 257 (1995); M. Quirós, "Constraints on the Higgs boson properties from the effective potential," hep-ph/9703412; T. Hambye and K. Riesselmann, Phys. Rev. D 55, 7255 (1997).

[12] H. Komatsu, Prog. Theor. Phys. 67, 1177 (1982); R. A. Flores and M. Sher, Ann. Phys. (N.Y.) 148, 95 (1983); M. Sher, Phys. Rep. 179, 273 (1989); D. Kominis and R. S. Chivukula, Phys. Lett. B 304, 152 (1993); S. Kanemura, T. Kubota, and E. Takasugi, ibid. 313, 155 (1993); S. Nie and M. Sher, ibid. 449, 89 (1999); A. Akeroyd, A. Arhrib, and E. Naimi, Phys. Lett. B 490, 119 (2000).

[13] S. Kanemura, T. Kasai, and Y. Okada, Phys. Lett. B 471, 182 (1999).

[14] J. Casas, V. Clemente, A. Ibarra, and M. Quirós, Phys. Rev. D 62, 053005 (2000).

[15] D. Zeppenfeld, R. Kinnunen, A. Nikitenko, and E. RichiterWas, Phys. Rev. D 62, 013009 (2000).

[16] E. Boos, J.-C. Brient, D. W. Reid, H. J. Schreiber, and R. Schanidze, Eur. Phys. J. C 19, 455 (2001); Rick van Kooten, talk given at the Berkeley Linear Collider Meeting, 2000, http://needmore.physics.indiana.edu/rickv/nlc/talks/ Berkeley_2000/Berkeley_2000.html.

[17] M. Melles, talk given at LCWS 2000 (Fermilab, 2000), hep-ph/0012195. 
[18] Z. Maki, M. Nakagawa, and S. Sakata, Prog. Theor. Phys. 28, 870 (1962).

[19] M. Ciuchini, G. Degrassi, P. Gambini, and G. F. Giudice, Nucl. Phys. B527, 21 (1998); P. Ciafaloni, A. Romanino, and A. Strumia, ibid. B524, 361 (1998); F. Borzumati and G. Greub, Phys. Rev. D 58, 074004 (1998); T. M. Aliev and E. O. Iltan, ibid. 58, 095014 (1998).

[20] H. E. Haber and H. E. Logan, Phys. Rev. D 62, 015011 (2000).

[21] J. F. Gunion, H. E. Haber, G. Kane, and Sally Dawson, The Higgs Hunter's Guide (Addison-Wesley, Reading, MA, 1990).

[22] ALEPH Collaboration, R. Barate et al., Phys. Lett. B 495, 1
(2000); L3 Collaboration, M. Acciarri et al., ibid. 495, 18 (2000).

[23] V. Drollinger and A. Sopczak, hep-ph/0102342.

[24] S. Kanemura, T. Kasai, G.-L. Lin, Y. Okada, J. J. Tseng, and C.-P. Yuan, hep-ph/0010233.

[25] M. Dima, talk given at LCWS 2000 (Fermilab, 2000), http:// www-lc.fnal.gov/lcws2000/; ALEPH Collaboration, R. Barate et al., Phys. Lett. B 407, 377 (1997).

[26] ALEPH Collaboration, R. Barate et al., Phys. Lett. B 487, 253 (2000). 